\title{
CLOVERLEAF REPRESENTATIONS OF SIMPLY CONNECTED 3-MANIFOLDS
}

\author{
BY
}

EDWIN E. MOISE

\begin{abstract}
Let $M$ be a triangulated 3-manifold satisfying the hypothesis of the Poincare Conjecture. In the present paper it is shown that there is a finite linear graph $K_{1}$ in the 3-sphere, with exactly two components, and a finite linear graph $K_{2}$ in $M$, such that when the components of the graphs $K_{i}$ are regarded as points, the resulting hyperspaces are homeomorphic. $K_{2}$ satisfies certain conditions which imply that each component of $K_{2}$ is contractible in $M$. Thus the conclusion of the theorem proved here is equivalent to the hypothesis of the Poincaré Conjecture.
\end{abstract}

1. Statement of results. All manifolds discussed in this paper will be piecewise linear. By a linear graph we mean either a 1-dimensional complex or a 1dimensional polyhedron, according to convenience in the context. By a loopgraph we mean a linear graph $L$, containing a point $P_{0}$ such that $L$ is the union of a finite collection of 1 -spheres $\left\{J_{1}, J_{2}, \cdots, J_{n}\right\}$ such that the intersection of every two of them is $P_{0}$. These 1-spheres are called the loops of $L$, and $P_{0}$ is called the center of $L$. Let $L$ be a loop-graph in a triangulated 3 manifold $M$. Suppose that there is a collection $\left\{D_{1}, D_{2}, \cdots, D_{n}\right\}$ of polyhedral disks, such that $\mathrm{Bd} D_{i}=J_{i}$ for each $i$, and such that the intersection of every two of the disks $D_{i}$ is $P_{0}$. Then $L$ is called a cloverleaf. (Here, as usual, if $D$ is an $m$-manifold with boundary, then $\operatorname{Bd} D$ is the boundary of $D$. The interior $D-\operatorname{Bd} D$ of $D$ will be denoted by Int $D$.) The set $\left\{D_{1}, D_{2}\right.$, $\left.\cdots, D_{n}\right\}$ is called $a$ set of spanning disks for $L$.

Now let $K=\bigcup_{i=1}^{n} I_{i}$ and $L=\bigcup_{i=1}^{n} J_{i}$ be cloverleaves, with the same number of loops, in the same triangulated 3-manifold $M$. Suppose that we can choose the order of the loops in $K$ and $L$, and choose spanning disks $\left\{D_{1}\right.$, $\left.D_{2}, \cdots, D_{n}\right\}$ for $K$, and spanning disks $\left\{E_{1}, E_{2}, \cdots, E_{n}\right\}$ for $L$, in such a way that (1) $D_{i}$ intersects $E_{j}$ only if $i=j$, and (2) $D_{i}$ and $E_{i}$ intersect in the same way, topologically, as two linked circular regions in Euclidean 3 space $\mathbf{R}^{3}$. Then $K$ and $L$ are simply linked.

Received by the editors February 3, 1972 and, in revised form, February 1, 1974. AMS (MOS) subject classifications (1970). Primary 55A40.

Key words and phrases. Hyperspaces of the 3-sphere, Poincaré Conjecture.

Copyright $\odot$ 1975, American Mathematical Society 
Let $K$ be a finite linear graph in a triangulated 3-manifold $M$. By a slight abuse of language, $M / K$ denotes the space whose points are the components of $K$ and the points of $M-K$ (with the usual topology).

THEOREM 1.1 (THE ClOVERLEAF THEOREM). Let $M$ be a compact, connected, simply connected triangulated 3-manifold. Then there is a linear graph $K_{1}$ in the 3-sphere $S^{3}$, and a linear graph $K_{2}$ in $M$, such that (1) either $K_{2}$ is empty or $K_{2}$ is the union of two simply linked cloverleaves, and (2) $S^{3} / K_{1}$ and $M / K_{2}$ are homeomorphic.

The following theorems throw some light on the cloverleaf theorem. Their proofs are easy, and are omitted.

THEOREM 1.2. Let $K$ be a finite linear graph in a connected triangulated 3-manifold $M$. If $M$ is simply connected, then so also is $M / K$.

TheOREM 1.3. Let $M$ and $K$ be as in Theorem 1.2, and suppose that the components of $K$ are cloverleaves. If $M / K$ is simply connected, then so also is $M$.

THEOREM 1.4. Let $M$ and $K$ be as in Theorems 1.2 and 1.3. Suppose that there is a linear graph $H$ in $S^{\mathbf{3}}$ such that $S^{\mathbf{3}} / H$ and $M / K$ are homeomorphic. Then $M$ is simply connected.

Theorem 1.4 is a corollary of the two preceding theorems. By Theorem $1.2, S^{3} / H$ is simply connected. Therefore so also is $M / K$. By Theorem 1.3 , $M$ is simply connected.

Theorem 1.4 indicates that it is not impossible a priori for the conclusion of Theorem 1.1 to be used as a working hypothesis for $M$ in a proof of the Poincaré Conjecture.

Without the hypothesis that $K$ is a finite linear graph, Theorem 1.2 becomes false. See Bing's announcement [B] that if $K$ is a solenoid in $S^{3}$, then $S^{3} / K$ is not simply connected.

Without the hypothesis that the components of $K$ are cloverleaves, Theorems 1.3 and 1.4 become false. To see this, let $M$ be a triangulated 3-manifold which is compact, connected, and orientable, but not simply connected. Let $K$ be the 1-skeleton of $M$, and let $N$ be a regular neighborhood of $K$. Then the closure $\mathrm{Cl}(M-N)$ is a tubular set, that is, it is homeomorphic to a regular neighborhood of a linear graph, with a certain 1-dimensional Betti number $p^{1}=$ $p^{1}(\mathrm{Cl}(M-N))$ (with integers modulo 2 as coefficients). Since $\mathrm{Cl}(M-N)$ cannot contain a "solid Klein bottle", it follows that the topology of $\mathrm{Cl}(M-N)$ is completely described by $p^{1}$. Evidently $M-K$ is homeomorphic to $M-N$, and $M / K$ is homeomorphic to the one-point compactification of $M-K$. 
But all this can be copied in $S^{3}$. Let $H$ be a linear graph in $S^{3}$, lying in a polyhedral 2-sphere, with $p^{1}(H)$ chosen so that $S^{3}-H$ is homeomorphic to $M-K$. Then $S^{3} / H$ is homeomorphic to the one-point compactification of $S^{3}-H$. Therefore, by Theorem $1.2, S^{3} / H$ is simply connected. Therefore, so also is $M / K$. But, at the outset, $M$ was not simply connected.

Thus the distinctive topology of a compact connected orientable triangulated 3-manifold is destroyed when we map its 1-skeleton onto a point. This observation is of course the first step in the proofs of deeper results of Lickorish [L] and Hempel $[\mathrm{H}]$.

2. Special monotonic mappings. The following was proved in $[\mathrm{M}]$.

THEOREM 2.1. Let $M$ be a triangulated 3-manifold, and suppose that $M$ is compact, connected, and simply connected. Then there is a subcomplex $K$ of a triangulation of the 3-sphere $S^{3}$, and a mapping $f: S^{3} \rightarrow M$ of $S^{3}$ onto $M$, such that

(1) $\operatorname{dim} K \leqslant 2$,

(2) $f \mid K$ is simplicial (relative to $K$ and a subdivision of $M$ ),

(3) $f \mid\left(S^{3}-K\right)$ is one-to-one,

(4) $f(K) \cap f\left(S^{3}-K\right)=0$,

(5) $f$ is monotonic, and

(6) each set $f^{-1}(x)$ is either a point or a linear graph.

We shall now modify the mapping $f$, strengthening some of its properties and weakening others; (6) will be lost.

First, we may suppose that the given triangulation $X$ of $S^{3}$ (in which $K$ forms a subcomplex) has the property that

(7) If $\sigma \in X$, then $\sigma \cap K$ either is empty or is a single face of $\sigma$.

(If the given $X$ does not have this property, then we form a subdivision of $X$, by introducing exactly one new vertex in the interior of each simplex $\sigma^{i}$ of $X(i>0)$ that does not lie in $K$. The new triangulation satisfies (7).)

Second, we may suppose that

(8) $K$ is the closure of the union of the nondegenerate sets $f^{-1}(x)(x \in M)$.

(If (8) does not hold, then $f$ IInt $\sigma$ is one-to-one, for some $\sigma \in K$. We can then delete Int $\sigma$ from $K$, preserving the stated properties of $K$.)

DEFINITION 2.1. By a cell-complex we mean a locally finite collection $\mathbf{C}$ of topological cells, such that (1) if $C \in \mathrm{C}$, then $\mathrm{Bd} C$ is a union of elements of C, (2) different sets Int $C$ are disjoint, and (3) if two elements of $\mathbf{C}$ intersect, then their intersection is the union of the elements of a subcollection of C. If $C_{1} \subset C_{2}$, then $C_{1}$ is a face of $C_{2}$ and a face of C. If $\operatorname{dim} C_{1}=i$, then $C_{1}$ is an i-face of $C_{2}$ (and of $\mathrm{C}$ ). $\mathrm{C}$ is called a cell-decomposition of the union $C^{*}$ of the elements of $C$. 
The images $f(\sigma)$ of the simplices of $X$ now form a cell-decomposition $f(X)$ of $M$, whose faces in various dimensions are the images of the faces of $X$. Evidently $f(X)$ forms a subcomplex of a triangulation $Y$ of $M$, and so the elements of $f(X)$ are polyhedral in $M$, relative to $Y$. Suppose that $K$ is not connected, and let $B$ be a polygonal arc in the 1-skeleton $X^{1}$, joining two vertices $v, v^{\prime}$ of different components of $K$, and intersecting $K$ only in $v$ and $v^{\prime}$. Then $f(B)$ is a polygonal arc in $M$. Therefore there is a mapping $\phi: M \rightarrow M$, such that (1) $\phi(f(B))$ is a point, (2) $\phi$ is a homeomorphism everywhere else, and (3) $\phi \mid f(K)$ is piecewise linear. Let $f^{\prime}=\phi(f)$, and let $K^{\prime}=$ $K \cup B$. Then $f^{\prime}$ and $K^{\prime}$ satisfy all the conditions for $f$ and $K$, except for (2); the images $f^{\prime}(\sigma)\left(\sigma \in K^{\prime}\right)$ are homeomorphic to simplices, but they are not necessarily simplices; in general, we have

$\left(2^{\prime}\right)$ For each $\sigma \in K^{\prime}, f^{\prime} \mid \sigma$ is a simplicial mapping $f \mid \sigma: \sigma \rightarrow \tau$, followed by a piecewise linear homeomorphism $\tau \rightarrow M$.

Geometrically, in $X=S^{3}$, the sets $\sigma^{2} \cap f^{\prime-1}\left(f^{\prime}(x)\right)\left(\sigma^{2} \in K\right)$ are the same as the sets $\sigma^{2} \cap f^{-1}(f(x))$. Note that, to preserve (7), we may need to subdivide $X$, by the same method as before.

In a finite number of such steps, we get $f^{\prime}$ and $K^{\prime}$ satisfying (1), (2), (3)-(8), and

(9) $K^{\prime}$ is connected.

Now let $L=f^{\prime}\left(K^{\prime}\right)$. Then $L$ forms a linear graph, in the strict sense of a complex, in which the edges and vertices are known; these are the images of the simplices of $K$, and $L$ forms a polyhedron in $M$. We want to produce a situation in which $L$ becomes the union $L^{\prime}$ of a finite number of polyhedral arcs, every two of which have the same endpoints. (See condition (2") below.) Note that $L$ is already connected, because $K^{\prime}$ is.

Step 1. Let $V$ be a subgraph of $L$, such that (a) $V$ is connected, (b) $V$ is acyclic, and (c) $V$ is maximal with respect to properties (a) and (b). Let $W$ be the closure of $L-V$. Then every edge of $W$ joins two vertices of $V$. Thus if $V$ is mapped onto a point, every edge of $W$ is mapped onto a 1-sphere.

Step 2. We now subdivide $X$ in the following way. Given $\sigma^{2}=v_{0} v_{1} v_{2} \in$ $K^{\prime}$, such that $f^{\prime}\left(\sigma^{2}\right)$ is an edge $f^{\prime}\left(v_{0} v_{1}\right)=f^{\prime}\left(v_{0} v_{2}\right)$ of $W$, we subdivide $\sigma^{2}$, using the midpoints of $v_{0} v_{1}$ and $v_{0} v_{2}$ as the only new vertices. This gives a subdivision $K^{\prime \prime}$ of $K^{\prime}$. In each $\sigma^{3}$ that contains such a $\sigma^{2}$, we introduce a new vertex $v$ in the interior, and subdivide $\sigma^{3}$ by forming the join of $v$ with the subdivison of $\mathrm{Bd} \sigma^{3}$ already defined. This gives a subdivision $X^{\prime}$ of $X$, in which $K^{\prime \prime}$ forms a subcomplex. As before, $f^{\prime}\left(X^{\prime}\right)$ forms a cell decomposition of $M$, and forms a subcomplex of a triangulation $Y^{\prime}$ of $M$.

Now $S^{\mathbf{3}}-K^{\prime \prime}$ is connected, because its homeomorphic image $f^{\prime}\left(S^{\mathbf{3}}-K^{\prime \prime}\right)$ is the complement of the linear graph $L$ in $M$. Let $N\left(K^{\prime \prime}\right)$ be the union of 
the simplices of $X^{\prime}$ that intersect $K^{\prime \prime}$, and let $T$ be the closure of $S^{3}-N\left(K^{\prime \prime}\right)$, so that $T$ forms a subcomplex of $X^{\prime}$. Then $T$ is connected. To see this, let $P$ and $Q$ be points of $T$, and let $P Q$ be a broken line in $S^{3}-K^{\prime \prime}$, joining $P$ to $Q$. Then $P Q$ can be forced off the simplices of $N\left(K^{\prime \prime}\right)$, one at a time; and this gives a path from $P$ to $Q$ in $T$. It follows that the 1-skeleton $T^{1}$ of $T$ is connected. Note also that $T^{1}$ contains all new vertices of $X^{\prime}$ in interiors of 3-simplices of $X$. It follows that there is a connected acyclic linear graph $J_{1}$ in $T^{1}$, containing all such new vertices. For each edge $e=f\left(\sigma^{2}\right)$ of $W$, we choose an edge $\sigma^{1}$ of $K^{\prime}$ such that $e=f^{\prime}\left(\sigma^{1}\right)$; and we let $v_{e}$ be the midpoint of $\sigma^{1}$, so that $v_{e}$ is a vertex of $X^{\prime}$. For each such $v_{e}$, we add to $J_{1}$ an edge of $X^{\prime}$ that joins $v_{e}$ to a point of $J_{1}$. This gives a connected acyclic linear graph $J_{2}$.

Finally, let $X^{\prime \prime}$ be a subdivision of $X^{\prime}$, such that every simplex of $X^{\prime \prime}$ intersects $K^{\prime \prime} \cup J_{2}$ in a simplex of $K^{\prime \prime} \cup J_{2}$ (or in the empty set). To do this, we use the same join-construction as before. Let $K^{\prime \prime \prime}=K \cup J_{2}$. As before, $f^{\prime}\left(X^{\prime \prime}\right)$ forms a cell decomposition of $M$, and there is a triangulation $Y^{\prime \prime}$ of $M$ in which $f^{\prime}\left(X^{\prime \prime}\right)$ forms a subcomplex.

Step 3. Now $V$ and $f^{\prime}\left(J_{2}\right)$ form subcomplexes of $Y^{\prime \prime}$, and each is a connected acyclic linear graph. Let $\psi: M \rightarrow M$ be a mapping such that $\psi(V)$ and $\psi\left(f^{\prime}\left(J_{2}\right)\right)$ are points, such that $\psi$ is a homeomorphism elsewhere, and such that each set $\psi\left(f^{\prime}(\sigma)\right)\left(\sigma \in X^{\prime \prime}\right)$ is a polyhedron. Thus $\psi\left(f^{\prime}\left(X^{\prime \prime}\right)\right)$ is a polyhedral cell decomposition of $M$, relative to the triangulation $Y^{\prime \prime}$. Let

$$
f^{\prime \prime}=\psi\left(f^{\prime}\right): S^{3} \rightarrow M .
$$

Then $f^{\prime \prime}\left(J_{2}\right)$ is a point, $f^{\prime \prime}\left(f^{\prime-1}(V)\right)$ is a point, and $K^{\prime \prime \prime}$ is the closure of the union of the nondegenerate sets $f^{\prime \prime-1}(P)(P \in M)$. Thus $f^{\prime \prime}, K^{\prime \prime}$, and $X^{\prime \prime}$ satisfy all the conditions for $f^{\prime}, K^{\prime}$, and $X^{\prime}$, and also the following:

(2") There are points $v, v^{\prime}$ of $M$ such that for each $\sigma^{2} \in K^{\prime \prime \prime}$, either $f^{\prime \prime}\left(\sigma^{2}\right)$ is a point or $f^{\prime \prime} \mid \sigma^{2}$ is a simplicial mapping $\sigma^{2} \rightarrow \tau^{1}$ followed by a piecewise linear homeomorphism $\tau^{1} \leftrightarrow B_{i}$, where $B_{i}$ is a broken line joining $v$ to $v^{\prime}$. Different sets $B_{i}$ intersect only at $v$ and $v^{\prime}$.

We also have:

(10) $f^{\prime \prime}\left(X^{\prime \prime}\right)$ forms a polyhedral cell-decomposition of $M$ (relative to the triangulation $\left.Y^{\prime \prime}\right)$.

If $f^{\prime \prime}$ and $K^{\prime \prime \prime}$ satisfy (1), (2"), (3)-(5), and (8)-(10), then $f^{\prime \prime}$ is called a special monotonic mapping. Note that (6) was lost, under the construction which gave us $\left(2^{\prime \prime}\right)$. These conditions can be reformulated more simply as follows. In the following definition, the given triangulation is understood to be the triangulation $Y^{\prime \prime}$ used at the end of the preceding discussion. $K^{\prime \prime \prime}, f^{\prime \prime}$, and $X^{\prime \prime}$ are replaced by $K, f$, and $X$ respectively. 
DEFinition 2.2. Let $M$ be a triangulated 3-manifold, let $X$ be a triangulation of $S^{3}$, let $f$ be a mapping of $S^{3}$ onto $M$, and let $K$ be the closure of the union of the nondegenerate sets $f^{-1}(P)(P \in M)$. Suppose that

(1) $K$ forms a subcomplex of $X$;

(2) $\operatorname{dim} K \leqslant 2$;

(3) Every simplex of $X$ intersects $K$ in a simplex (or in the empty set);

(4) There are points $v, v^{\prime}$ of $M$ such that for each $\sigma^{2} \in K$, either $f\left(\sigma^{2}\right)$ is a point or $f \mid \sigma^{2}$ is a simplicial mapping $\sigma^{2} \rightarrow \tau^{1}$ followed by a piecewise linear homeomorphism $\tau^{1} \leftrightarrow B_{i}$, where $B_{i}$ is a broken line joining $v$ to $v^{\prime}$. Different sets $B_{i}$ intersect only at $v$ and $v^{\prime}$;

(5) $f$ is monotonic; and

(6) the images $f(\sigma)$ of the simplices of $X$ form a polyhedral cell decomposition of $M$.

Then $f$ is a special monotonic mapping (relative to $X$ ).

The preceding discussion has proved the following:

THEOREM 2.2. Let $M$ be a triangulated 3-manifold, and suppose that $M$ is compact, connected, and simply connected. Then $S^{\mathbf{3}}$ and $M$ can be triangulated in such a way that $M$ is the image of $S^{\mathbf{3}}$ under a special monotonic mapping.

Hereafter we shall have no occasion to use Theorem 2.1.

The loss of condition (6) of Theorem 2.1 is not a serious matter; we shall get it back, at the end of the argument, by the unknotting process in $\S 12$ of [M].

3. An outline of the rest of the proof. The logical apparatus used in the proof of the cloverleaf theorem is tedious, and the technical definitions in later sections may be easier to understand if we first give a heuristic sketch, indicating the problems that they are designed to deal with.

Given a special monotonic mapping $f: S^{3} \rightarrow M$, let $G=\left\{f^{-1}(y) \mid y \in M\right\}$, let $S^{3} / G$ be the resulting hyperspace, and let $\pi$ be the projection $S^{3} \rightarrow S^{3} / G$. Let $G^{\prime}=M$, and let $\pi^{\prime}$ be the projection $M \rightarrow M / G^{\prime}$. Let $y$ be a point of a set Int $B_{i}$. Then $f^{-1}(y)$ is a linear graph. Such a set $f^{-1}(y)$ will be called a generic element of $G$. Each edge $e_{i}$ of $f^{-1}(y)$ lies in a 2-simplex of the "singularity complex" $K$. Suppose, for the moment, that we are dealing with the simplest of the nontrivial cases, namely, Bing's example, described in $[M]$, so that $f^{-1}(y)$ is a figure 8 , and $f(K)$ is a linear segment $B$. Let $\sigma^{2}=v_{0} v_{1} v_{2}$ be a 2-simplex of $K$, such that $f\left(\sigma^{2}\right)=f\left(v_{0} v_{1}\right)=f\left(v_{0} v_{2}\right)$, an edge of $f(K)$. In the figure on the left below, the sets $\sigma^{2} \cap f^{-1}(y)(y \in \operatorname{Int} B)$ are horizontal segments. $B$ is shown on the right. 


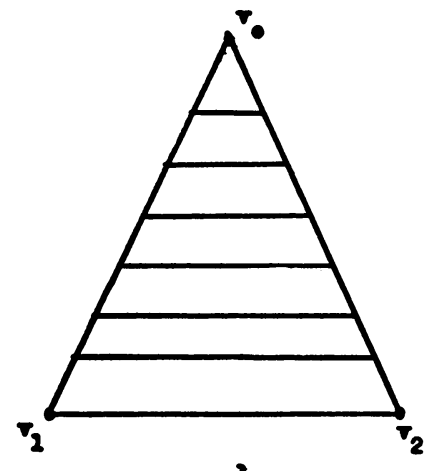

In $8^{3}$

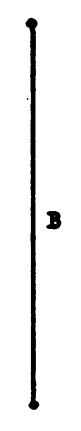

In $x$

FIGURE 1

If the sets $f^{-1}(y)(y \in \operatorname{Int} B)$ are acyclic, then the cloverleaf theorem follows easily, and it also follows that $M$ is a 3-sphere. (See Finney [F].) If the sets $f^{-1}(y)$ are not acyclic, then $\sigma^{2}$ can be chosen so that each set $f^{-1}(y)-f^{-1}(y) \cap \sigma^{2}(y \in \operatorname{Int} B)$ is connected. (In Bing's example, every 2 simplex of $K$ has this property.) Hereafter, we shall assume that $\sigma^{2}$ satisfies this condition.

Step 1. First we split $v_{1} v_{2}$ into two arcs $e_{1}, e_{2}$, each of which projects onto a point, in a new hyperspace $S^{3} / G_{1}$ of $S^{3}$, as shown in Figure 2.

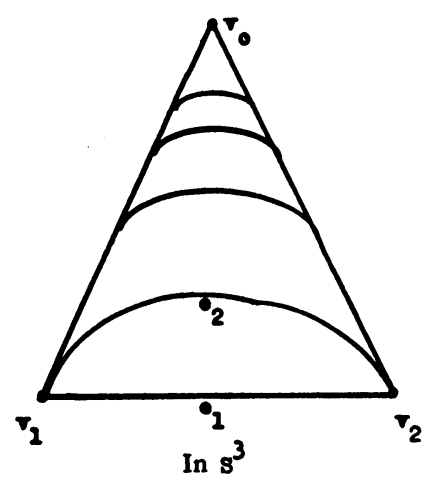

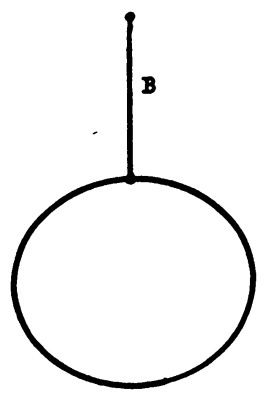

In $x$

Figure 2

To copy this operation in $M$, so as to preserve the homeomorphism between the hyperspaces, we introduce a polyhedral 1-sphere $J$ in $M$, spanning a polyhedral disk, and map $J$ onto a point, getting a new hyperspace $M / G_{1}^{\prime}$ of $M . J$ intersects $B$ in a point, as shown in the figure on the right above.

In later steps, $\pi_{i}$ and $\pi_{i}^{\prime}$ will denote the projections $S^{3} \rightarrow S^{3} / G_{i}$ and 
$M \rightarrow M / G_{i}^{\prime} \quad K_{i}$ and $K_{i}^{\prime}$ will denote the "singularity complexes" in $S^{3}$ and $M$, that is, the closures of the unions of the nondegenerate elements of $G_{i}$ and $G_{i}^{\prime}$. From now on, $K_{i}^{\prime}$ will be nonempty.

Step 2, Next, in $S^{3}$, we introduce a polyhedral disk $D_{1}$ mapped onto the same linear interval as $\sigma^{2}$, in a new hyperspace, so that $D_{1} \cup \sigma^{2}$ looks like a portion of the configuration used in Bing's example. (Here, and hereafter in this section, when we "change" geometric configurations, we do not assign new names to the new sets thus obtained.) We now have a new hyperspace $S^{3} / G_{2}$ of $S^{3}$, as shown in Figure 3.
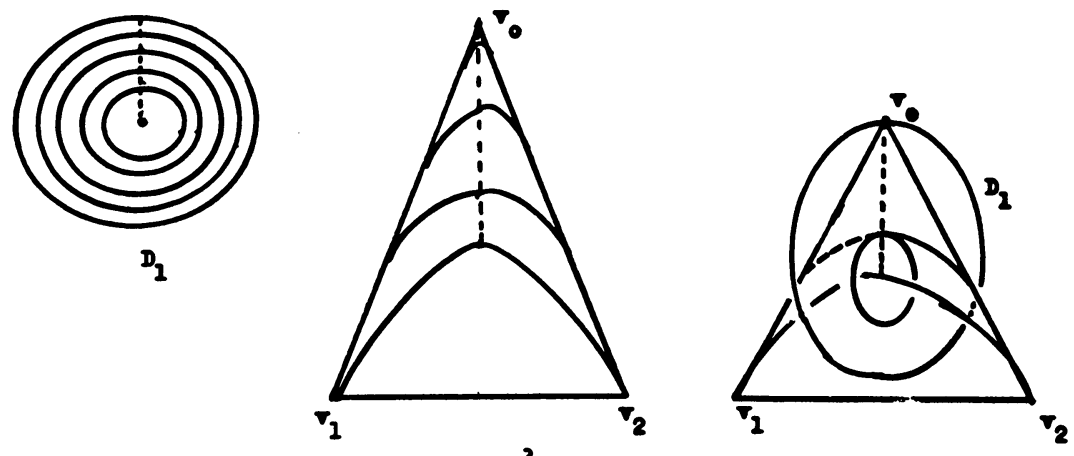

111 in $8^{3}$

Figure 3

We copy this operation in $M$, by introducing a disk $D_{2}$ linked with $J$ as in the figure below; and we define a new $M / G_{2}^{\prime}$ in which $\pi_{2}^{\prime}\left(D_{2}\right)=\pi_{2}^{\prime}(B)$. In Figure 4, $I=\operatorname{Bd} D_{2}$.

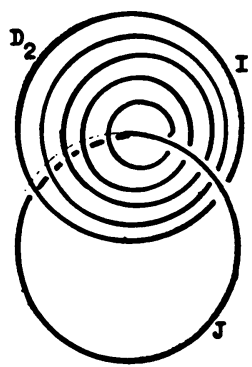

FIGURE 4

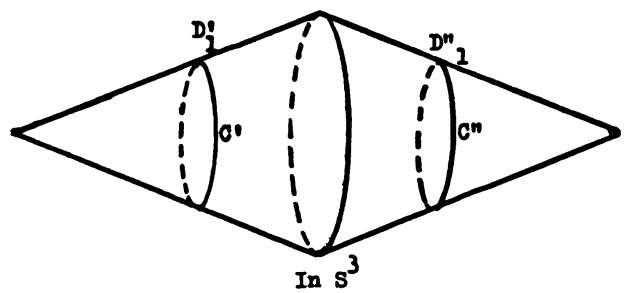

FIGURE 5

Step 3. Next we simplify $S^{3} / G_{2}$ (or rather $G_{2}$ ) by the operation used in the author's proof (presented in [M]) that Bing's hyperspace is homeomorphic to $S^{3}$. That is, we split $D_{1}$ apart, along Int $D_{1}$, into two polyhedral 2-cells 
$D_{1}^{\prime}, D_{1}^{\prime \prime}$, bounding a polyhedral 3-cell $C$. In the new hyperspace, we regard Int $C$ as empty. See Figure 5. This operation induces a homeomorphism of $S^{3} / G_{2}$; the point is that not only in Bing's example, but also in general, it does no harm to split a circle $c$ in $D_{1}$ into two circles $c^{\prime}$ and $c^{\prime \prime}$, because $c^{\prime}$ and $c^{\prime \prime}$ always lie in the same element of the resulting upper semicontinuous decomposition $G_{3}$ of $S^{3}-$ Int $C$. In the general case, this is a consequence of the initial hypothesis that for each generic set $f^{-1}(y)$, the set $f^{-1}(y)-f^{-1}(y) \cap \sigma^{2}$ is connected. Thus $\left(S^{3}-\right.$ Int $\left.C\right) / G_{3}$ is homeomorphic to $S^{3} / G_{2}$, and hence to $M / G_{2}^{\prime}$. Let $G_{3}^{\prime}=G_{2}^{\prime}$.

Step 4. We now map $C$ onto the union of two linear intervals, by a mapping which is a homeomorphism on $S^{3}-C$, in such a way that the latitudinal circles $c^{\prime}$ and $c^{\prime \prime}$ are mashed onto points. We now have hyperspaces $S^{3} / G_{4}$ and $M / G_{4}^{\prime}=M / G_{3}^{\prime}$. The local situation in $S^{3}$ is shown in Figure 6. The situation in $M$ is still as in Figure 4.

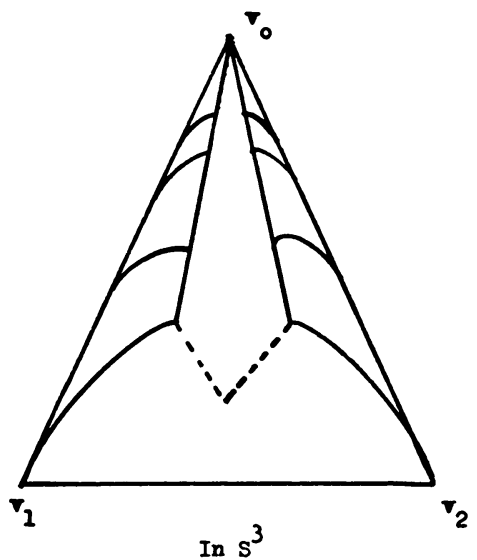

Figure 6

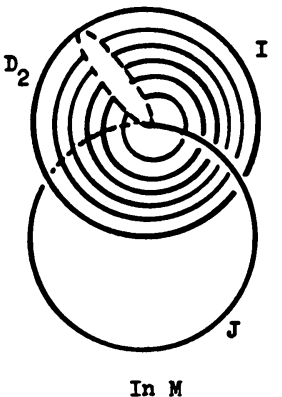

FigURE 7

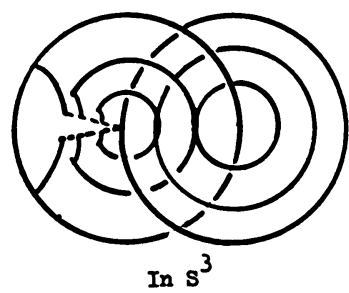

FIGURE 8

Here the quadrilateral in the middle of the figure shows the place where Figure 5 used to be.

Step 5. In $M / G_{4}^{\prime}, \pi_{4}^{\prime}\left(D_{2}\right)$ is a linear interval, and separates space locally, in its interior. That is, $\pi_{4}^{\prime}\left(D_{2}\right)$ is the intersection of two 3-cells, each of which contains the interior of the edge in its interior. Since $S^{3} / G_{4}$ and $M / G_{4}^{\prime}$ are homeomorphic, $\pi_{4}\left(v_{0} v_{1}\right)$ must have the same property. This property of $M / G_{4}^{\prime}$ can be abolished by splitting $D_{2}$ along the interior of a radial edge, as shown in Figure 7. It is a fact that this operation can be copied in $S^{3}$, by splitting $K_{4}$ apart in some way. In Bing's example, this is immediately plausible. The situation in $S^{3}$ is as in Figure 8. The disk on the left has already been split. To copy in 
$S^{3}$ the splitting of $M$, we split the disk on the right, along any radial edge. Thus we have homeomorphic hyperspaces $S^{3} / G_{5}$ and $M / G_{5}^{\prime}$.

Step 6. In Bing's example, the nondegenerate elements of both $G_{5}$ and $G_{5}^{\prime}$ now consist of two simply linked circles (that is, two simply linked cloverleaves with one loop apiece), plus a continuous family of generic sets which are acyclic linear graphs. The latter can be shrunk to points by operations preserving the topologies of the hyperspaces. (See, for example, the operation $\alpha^{\prime}$, defined on p. 463 of [M].) This gives homeomorphic hyperspaces, each of which looks like Figure 9.

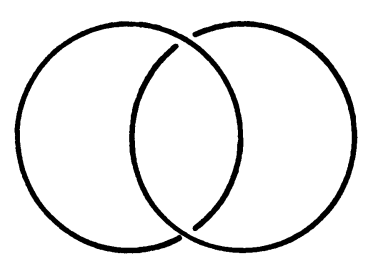

FiguRE 9

In the general case, we can still get two simply linked circles in $M$, by shrinking the generic sets; and by the same operations in $S^{3}$, we can get rid of the "free endpoints" of the corresponding generic sets. But we normally expect that there will still be 2-simplices in the resulting singularity complex $K_{6}$ which are mapped onto $B$ by the homeomorphism between the hyperspaces.

Step 7. The next step, then, is to deform $B$ so as to isolate it from the two 1-spheres in $G_{6}^{\prime}$ and from the disks that they bound (except at the endpoints). See Figure 10.

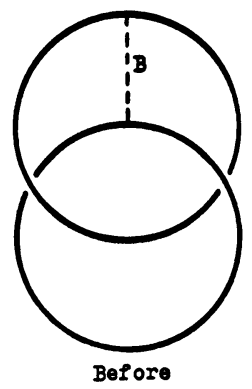

In $M$

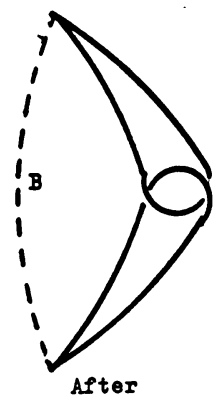

\section{Figure 10}

We now repeat Steps 1 through 6 , keeping clear of the disks on the right. The main stages in $M$ are shown in Figure 11. The corresponding figures for the 
operations in $S^{3}$ would be exactly the same as before.
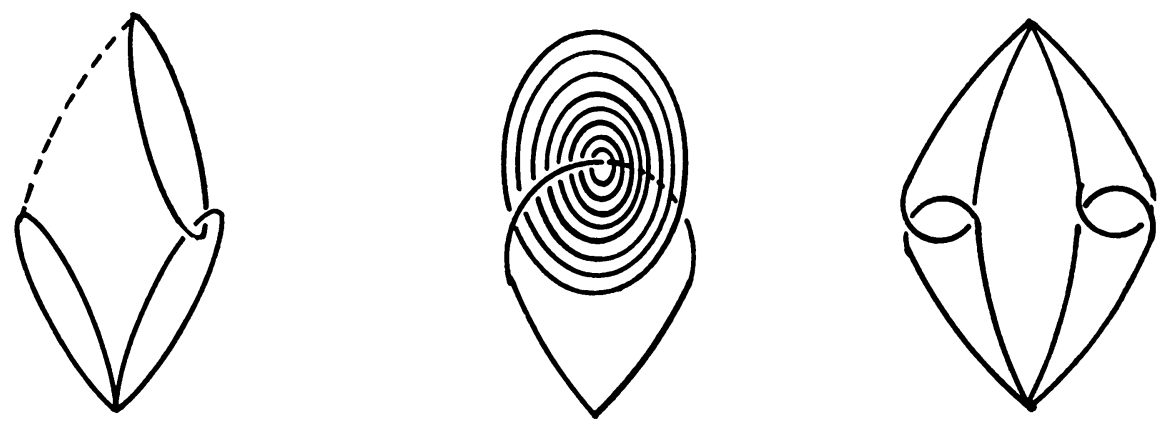

\section{FIGURE 11}

And, of course, in the general case, we have more than one $B$ to deal with. But the process must terminate, because it reduces the 1-dimensional Betti numbers of the generic sets $\pi_{i}^{-1}(y)$.

The general character of the technical problems should now be clear.

(I) The sets and mappings that we use are "built with the hands," and so, in the last resort, there cannot be problems arising from wild imbedding. But in Step 2, we need for the disk $D_{2}$ to be the image of $D_{1}$, and we need for $D_{2}$ to be a polyhedron in $M$. Since a special monotonic mapping is never piecewise linear, even at the outset, except in trivial cases, we cannot simply claim that the image of a polyhedron in $S^{3}$ is a polyhedron in $M$. Therefore we need a "sufficiently dense" family of 2-dimensional polyhedra in $S^{3}$ whose images in $M$ are polyhedra; and we need for this relationship to be defined in such a way that it is preserved under iterations of Steps 1 through 7.

(II) In the general case, the splitting operation in $S^{3}$, described in Step 5, does not take the simple form that it takes in Bing's example; we may need not only to split an edge of $K_{4}$ into two edges, but also to split a 2-simplex of $K_{4}$ into two 2-simplices. (See $\S 4$ below.) These duplicate 2-simplices are not always eliminated in Step 6, and so, in general, $K_{5}$ and $K_{6}$ are not complexes. Therefore, to use a recursion argument, we need an apparatus in which $K$ is not required to be a complex.

(III) We need to assign a meaning to Step 7. Topologically speaking, the situations shown on the left and right in Figure 10 are indistinguishable. Much the same problem of logical definition already arises in Step 1, where we need to assign a meaning to the relationship suggested by Figure 2 .

Hence the apparatus described in the following sections. A word of warning: in the deductive form of the proof, the order of the steps will be changed, for technical reasons; Step 7 will be postponed until all iterations of the preceding 
steps have been carried out. Thus Problem (III) will be avoided rather than solved.

4. Shrink-equivalences of pseudo-simplicial hyperspaces. If $f: S^{3} \rightarrow M$ is a special monotonic mapping, and $G=\left\{f^{-1}(x) \mid x \in M\right\}$, then the pair $\left[S^{3}, G\right]$ will be called a pseudo-simplicial hyperspace of $S^{3}$. We shall need this idea in a more general form, in which (1) $S^{3}$ is allowed to be any compact connected triangulated 3-manifold $M$, (2) the hyperspace $M / G$ is not required to be a manifold, and (3) the mapping $\pi \mid K$ (where $\pi$ is the projection $M \rightarrow M / G$ and $K$ is the closure of the union of the nondegenerate sets $\left.\pi^{-1}(x)\right)$ is almost simplicial, but not quite.

DEFINITION 4.1. Let $\mathbf{C}$ be a cell complex, in a triangulated 3-manifold, such that the elements of $\mathbf{C}$ are polyhedra. Then $\mathbf{C}$ is a pseudo-simplicial cell complex, or a PS cell complex, and is a PS decomposition of the union $\mathbf{C}^{*}$ of its elements. Let $\mathbf{C}_{1}$ and $\mathbf{C}_{2}$ be PS cell complexes, and let $\phi$ be a homeomorphism $\mathbf{C}_{1}^{*} \leftrightarrow \mathbf{C}_{2}^{*}$. If $\phi\left(\mathbf{C}_{1}\right)=\mathbf{C}_{2}$ (that is, if $\phi$ maps elements of $\mathbf{C}_{1}$ onto elements of $\mathbf{C}_{2}$ ), then $\phi$ is pseudo-simplicial.

Note that while a PS cell decomposition is always a collection of polyhedra, the system $\mathbf{C}$ has not been assigned a linear structure, and so a pseudo-simplicial homeomorphism need not be piecewise linear.

Let $K$ be a finite cell-complex, in a finite complex $M$, such that the elements of $K$ are polyhedra in $M$. Let $K^{*}$ be the union of the elements of $K$. Let $\rho$ be a mapping $K^{*} \rightarrow L^{*}$, where $L$ is a finite complex, such that, for each $\sigma \in K, \rho \mid \sigma$ is a piecewise linear homeomorphism of $\sigma$ onto a simplex $\tau$ of $L$. Then the pair $[K, \rho]$ is called a skew-complex. If $\left[K_{1}, \rho_{1}\right]$ and $\left[K_{2}, \rho_{2}\right]$ are skew-complexes, then a mapping $f: K_{1}^{*} \rightarrow K_{2}^{*}$ is skew-simplicial if (1) if $\sigma_{1} \in K_{1}$, then $f\left(\sigma_{1}\right)=\sigma_{2} \in K_{2}$, and (2) for each $\sigma_{1} \in K_{1}, \sigma_{2}=$ $f\left(\sigma_{1}\right)$, the mapping

$$
\rho_{2} f\left(\rho_{1} \mid \sigma_{1}\right)^{-1}: \tau_{1} \rightarrow \tau_{2}=\rho_{2}\left(\sigma_{2}\right)
$$

is simplicial.

Thus a skew-complex resembles a complex, in that (1) its simplices look, combinatorially, like simplices, and (2) each simplex $\sigma$ has a linear structure, induced by $(\rho \mid \sigma)^{-1}$, relative to which skew-simplicial mappings are defined. Note, however, that a simplex is not determined when its vertices are named. Thus the set $\mathbf{S}$ of all skew-complexes is closed under the operation of "splitting the complex into two parts, combinatorially, along the interior of a simplex." For example, suppose that $[K, \rho]$ is a trivial skew-complex, in which $K$ is a single 2-simplex $\sigma^{2}$ (plus its faces) and $\rho$ is the identity. We can get a new skewcomplex $\left[K^{\prime}, \rho^{\prime}\right]$ by replacing $\sigma^{2}$ by two PL 2-cells $\sigma_{1}^{2}, \sigma_{2}^{2}$, with $\sigma_{1}^{2} \cap \sigma_{2}^{2}=$ $\operatorname{Bd} \sigma_{1}^{2} \cap \operatorname{Bd} \sigma_{2}^{2}=\mathrm{Bd} \sigma^{2}$. We then define $\rho^{\prime}$ so that, for $i=1,2, \rho^{\prime} \mid \sigma_{i}^{2}$ is a piecewise linear homeomorphism (PLH) $\sigma_{i}^{2} \leftrightarrow \sigma^{2}$ and $\rho^{\prime} \mid \mathrm{Bd} \sigma_{i}^{2}$ is the identity.

More generally, suppose that the elements of $K$ are PL cells in a PL 3 - 
manifold $M$, and let $\rho$ and $L$ be arbitrary. Let $D_{1}^{3}$ and $D_{2}^{3}$ be PL 3-cells in $M$, with

$$
D_{1}^{3} \cap D_{2}^{3}=\operatorname{Bd} D_{1}^{3} \cap \operatorname{Bd} D_{2}^{3}=D^{2},
$$

where $D^{2}$ is a 2-cell, and $D^{2} \cap K^{*}$ is a union of elements of $K$. For $i=$ 1,2 let $E_{i}=\mathrm{Cl}\left(\mathrm{Bd} D_{i}^{3}-D^{2}\right)$. Then for $i=1,2$ there is a PLH

$$
f_{i}: D_{i}^{3} \rightarrow D_{i}^{3}-\operatorname{Int} D^{2},
$$

such that $f_{i} \mid E_{i}$ is the identity. The operation which replaces $M$ by the polyhedron

$$
M^{\prime}=\left[M-\left(D_{1}^{3} \cup D_{2}^{3}\right)\right] \cup f_{1}\left(D_{1}^{3}\right) \cup f_{2}\left(D_{2}^{3}\right)
$$

will be called a splitting of $M$ at $D^{2}$. Evidently there is a PL mapping $g: M^{\prime} \rightarrow$ $M$, such that $g \mid f_{i}\left(D_{i}^{3}\right)=f_{i}^{-1}$ and such that $g$ is the identity elsewhere. Let $K^{\prime}$ be the set of all cells $\tau$ such that $g(\tau) \in K$. Now define $\rho^{\prime}: K^{\prime *} \rightarrow L$ by the condition $\rho^{\prime} \mid \tau=\rho(g \mid \tau)$. Then $\left[K^{\prime}, \rho^{\prime}\right]$ is a skew-complex. The operation which replaces $[K, \rho]$ by $\left[K^{\prime}, \rho^{\prime}\right]$ is called a splitting of $[K, \rho]$ at $K^{*} \cap \operatorname{Int} D^{2}$.

Similarly, consider a collection $K=\left\{v, v^{\prime}, B_{1}, B_{2}, \cdots, B_{n}\right\}$, where $v$ and $v^{\prime}$ are points, and the $B_{i}$ 's , are broken lines from $v$ to $v^{\prime}$, not intersecting elsewhere. Then $K$ forms a skew-complex, relative to an obvious mapping $\rho$ : $K^{*} \rightarrow \sigma^{1}$, where $\sigma^{1}$ is fixed and each mapping $\rho \mid B_{i}$ is a PLH.

By abuse of language, we may speak of a PS cell-complex as a skew-complex. if it is clear what sort of mapping $\rho$ is intended.

We can now generalize our preliminary definition of a pseudo-simplicial hyperspace.

DEFINITION 4.2. Let $M$ be a compact connected triangulated 3-manifold, let $\mathrm{C}$ be a pseudo-simplicial cell-decomposition of $M$, let $G$ be an upper semicontinuous decomposition of $M$, and let $\pi$ be the projection $M \rightarrow M / G$. Let $K$ be the closure of the union of the nondegenerate elements of $G$. Suppose that

(1) $K$ forms a skew-complex in $M$;

(2) $\operatorname{dim} K \leqslant 2$;

(3) if $C \in \mathrm{C}$, and $C$ intersects $K$, then $C \cap K$ forms a subcomplex both of $\mathrm{C}$ and of $K$;

(4) each $C \in \mathrm{C}$ intersects each element of $G$ in a contractible set;

(5) there is a triangulation $Y$ of $M / G$ such that $\pi(C)$ is a PS cell-decomposition $\mathbf{C}^{\prime}$ of $M / G$;

(6) $\pi(K)$ forms a skew-complex in $Y$ (with at most two vertices);

(7) $\pi \mid K$ is skew-simplicial; and

(8) $\pi$ is monotonic. 
Then the pair $[M, G]$ is a PS hyperspace of $M$ (with respect to $\mathbf{C}$ and Y).

Note that we do not call $M / G$ a PS hyperspace, because in the applications we shall be dealing with the structure of $G$, and this is not determined when $M$ and $M / G$ are known. For example, in Bing's example, $S^{3} / G$ is homeomorphic to $S^{3}$, and therefore homeomorphic to $S^{3} / S^{3}$; but $G$ and $S^{3}$ are different.

Note that $\mathrm{C}$ and $Y$ are not part of the structure of the pseudo-simplicial hyperspace $[M, G]$; we are merely requiring that such objects exist. The projection $M \rightarrow M / G$ will always be denoted by $\pi$, and $\mathbf{C}^{\prime}$ will always denote $\pi(\mathbf{C})$.

Evidently we have been generalizing the definition of a special monotonic mapping, and so we have immediately:

THEOREM 4.1. If $f: S^{3} \rightarrow M$ is a special monotonic mapping, and $G=$ $\left\{f^{-1}(x) \mid x \in M\right\}$, then $\left[S^{3}, G\right]$ is a PS hyperspace of $S^{3}$.

For $i=1,2$, let $\left[M_{i}, G_{i}\right]$ be a PS hyperspace of $M_{i}$, with respect to $\mathrm{C}_{i}$, and let $\phi$ be a homeomorphism $M_{1} / G_{1} \leftrightarrow M_{2} / G_{2}$. If $\mathrm{C}_{1}$ and $\mathrm{C}_{2}$ can be chosen so that $\phi\left(\mathbf{C}_{1}^{\prime}\right)=\mathrm{C}_{2}^{\prime}$, then $\phi$ is a shrink-equivalence between $\left[M_{1}, G_{1}\right]$ and $\left[M_{2}, G_{2}\right]$. If such a $\phi$ exists, then $\left[M_{1}, G_{1}\right]$ and $\left[M_{2}, G_{2}\right]$ are shrinkequivalent.

THEOREM 4.2. If $f: S^{3} \rightarrow M$ is a special monotonic mapping, then $\left[S^{3}, G\right]$ and $[M, M]$ are shrink-equivalent.

Proof. Let $\pi$ be the projection $S^{3} \rightarrow S^{3} / G$, and let $\phi(\pi(x))=f(x)$.

THEOREM 4.3. Let $\phi$ be a shrink-equivalence between $\left[M_{1}, G_{1}\right]$ and $\left[M_{2}, G_{2}\right]$, with respect to $\mathbf{C}_{1}$ and $\mathbf{C}_{2}$. Let $B_{2}$ be an edge of $\mathbf{C}_{2}$, such that $K_{2} \cap$ Int $B_{2}=0$. Then there is a shrink-equivalence $\phi^{\prime \prime}$, between $\left[M_{1}, G_{1}\right]$ and $\left[M_{2}, G_{2}\right]$, with respect to subdivisions $\mathbf{C}_{1,1}$ and $\mathbf{C}_{2,1}$ of $\mathbf{C}_{1}$ and $\mathbf{C}_{2}$ respectively, such that if $X_{2}$ is the union of the 3-cells of $C_{2,1}$ that have $B_{2}$ as an edge, then $X_{2}$ is a 3-cell and $\pi_{2} \mid X_{2}$ is a homeomorphism.

Proof. Let $C_{2, i}^{2}$ be a 2-cell of $C_{2}$, having $B_{2}$ as an edge. Let $P_{2}$ and $Q_{2}$ be the endpoints of $B_{2}$. Let $B_{2, i}$ be a polyhedral arc from $P_{2}$ to $Q_{2}$ in $C_{2, i}^{2}$, intersecting $\mathrm{Bd} C_{2, i}^{2}$ only in $P_{2}$ and $Q_{2}$. As always, let $P_{2}^{\prime}$ and $Q_{2}^{\prime}$ be $\pi_{2}\left(P_{2}\right)$ and $\pi_{2}\left(Q_{2}\right)$. Let $C_{1, i .}^{2}$ be the element of $C_{1}$ such that

$$
\phi\left(\pi_{1}\left(C_{1, i}^{2}\right)\right)=\pi_{2}\left(C_{2, i}^{2}\right) .
$$

Then there are vertices $P_{1, i}$ and $Q_{1, i}$ such that

$$
\phi\left(P_{1, i}^{\prime}\right)=P_{2}^{\prime} \quad \text { and } \phi\left(Q_{1, i}^{\prime}\right)=Q_{2}^{\prime}
$$


(Note the double subscripts: $P_{1, i}$ depends, in general, on $i$ as well as on $P_{2}$, and similarly for $Q_{1, i}$.) Let $B_{1, i}$ be a polygonal arc in $C_{1, i}^{2}$, from $P_{1, i}$ to $Q_{1, i}$, intersecting $\mathrm{Bd} C_{1, i}^{2}$ only in $P_{1, i}$ and $Q_{1, i}$.

There is now a shrink-equivalence

$$
\begin{aligned}
& \phi^{\prime}: M_{1} / G_{1} \leftrightarrow M_{2} / G_{2}, \\
& : \mathbf{C}_{1}^{\prime} \rightarrow \mathbf{C}_{2}^{\prime},
\end{aligned}
$$

such that

$$
\phi^{\prime}\left(P_{1, i}^{\prime}\right)=P_{2}^{\prime}, \quad \phi^{\prime}\left(Q_{1, i}^{\prime}\right)=Q_{2}^{\prime}, \quad \text { and } \quad \phi^{\prime}\left(B_{1, i}^{\prime}\right)=B_{2, i}^{\prime} .
$$

To construct such a $\phi^{\prime}$, first define $\phi^{\prime}=\phi$ on every 2-cell of $C_{2}^{\prime}$ other than the cells $\pi_{1}\left(C_{1, i}^{2}\right)$, on $\phi^{-1}\left(B_{2}^{\prime}\right)$, and in the interior of every 3-cell of $C_{1}^{\prime}$ that does not contain a cell $\pi_{1}\left(C_{1, i}^{2}\right)$. Then extend $\phi^{\prime}$ so that $\phi^{\prime}\left(B_{1, i}^{\prime}\right)=B_{2, i}^{\prime}$ for each $i$. Now $B_{1, i}^{\prime}$ separates $C_{1, i}^{2}$ into 2-cells, on whose boundaries $\phi^{\prime}$ is already defined. Similarly for the sets $B_{2, i}^{\prime} \subset \pi_{2}\left(C_{2, i}^{2}\right)$. Now extend $\phi^{\prime}$ so that

$$
\phi^{\prime}\left(\pi_{1}\left(C_{1, i}^{2}\right)\right)=\pi_{2}\left(C_{2, i}^{2}\right) \text {. }
$$

Finally, extend $\phi^{\prime}$ to the interiors of the 3-cells $C_{1, i}^{\prime}$ such that $\phi\left(C_{1, i}^{\prime}\right)$ is a 3-cell containing the cell $\pi_{2}\left(C_{2, i}^{2}\right)$.

Now consider a 3-cell $C_{1, i}$ of $\mathrm{C}_{1}$, such that $C_{1, i}^{\prime}$ is of this last type, and let $C_{2, i} \in \mathrm{C}_{2}$ be such that $\phi^{\prime}\left(C_{1, i}^{\prime}\right)=C_{2, i}^{\prime}$. Let $C_{2, i}^{2}$ and $C_{2, i+1}^{2}$ be the 2-faces of $C_{2, i}$ that contain $B_{2}$. Then $B_{2, i} \cup B_{2, i+1}$ is a (simple closed) polygon $J_{2, i}$ in $\mathrm{Bd} C_{2, i}$, and bounds a polyhedral disk $D_{2, i}$ in $C_{2, i}$ such that

$$
D_{2, i} \cap \mathrm{Bd} C_{2, i}=\mathrm{Bd} D_{2, i}
$$

Under our hypothesis, $\pi_{2} \mid D_{2, i}$ is a homeomorphism. Let $\sigma_{2, i}$ be the closure of the component of $C_{2, i}-D_{2, i}$ whose boundary contains $B_{2}$. Then $\pi_{2} \mid \sigma_{2, i}$ is also a homeomorphism.

We need to copy this pattern in $M_{1}$. In $C_{1, i}$, the endpoints $P_{1, i}$ and $P_{1, i+1}$ are not necessarily the same. But $P_{1, i}^{\prime}=P_{1, i+1}^{\prime}$; and since each element of $G_{1}$ intersects $\mathrm{Bd} C_{1}$ in a contractible set, it follows that $\pi_{1}^{-1}\left(P_{1, i}^{\prime}\right) \cap \mathrm{Bd} C_{1}$ is connected. Since $P_{1, i}$ and $P_{1, i+1}$ are vertices, it also follows that $P_{1, i}$ can be joined to $P_{1, i+1}$ by a polygonal arc $P_{1, i} P_{1, i+1}$ lying in $K^{1} \cap \mathrm{Bd} C_{1}$. Similarly for $Q_{1, i}$ and $Q_{1, i+1}$. Now let

$$
J_{1, i}=B_{1, i} \cup B_{1, i+1} \cup P_{1, i} P_{1, i+1} \cup Q_{1, i} Q_{1, i+1} \text {. }
$$

Now $J_{1, i}$ is the boundary of a polyhedral 2-cell $D_{1, i}$ such that Int $D_{1, i} \subset$ Int $C_{1, i}$, and $D_{1, i}^{\prime}$ is a 2-cell. (The projection $\pi_{1}$ merely shrinks to points each of two disjoint arcs in $\operatorname{Bd} D_{1, i}$, and is a homeomorphism elsewhere.) 
Finally, we define a new shrink-equivalence $\phi^{\prime \prime}$, such that $\phi^{\prime \prime}\left(D_{1, i}^{\prime}\right)=D_{2, i}^{\prime}$ for each $i$. First we define $\phi^{\prime \prime}=\phi$ on the set $M-\cup_{i}$ Int $C_{1, i}$. Then we extend the given $\phi^{\prime \prime} \mid \mathrm{Bd} D_{1, i}^{\prime}$ to $D_{1, i}^{\prime}$, so that $\phi^{\prime \prime}\left(D_{1, i}^{\prime}\right)=D_{2, i}^{\prime}$. Finally we observe that $D_{1, i}^{\prime}$ decomposes $C_{1, i}^{\prime}$ into two 3-cells, intersecting in $D_{1, i}^{\prime}$; and similarly for $D_{2, i}^{\prime}$ in $C_{2, i}^{\prime}$. Now extend $\phi^{\prime \prime}$ to the interiors of these 3-cells, in such a way that $\phi^{\prime \prime}\left(\sigma_{1, i}^{\prime}\right)=\sigma_{2, i}^{\prime}$, where $\sigma_{1, i}$ is one of the 3-cells into which $D_{1, i}$ decomposes $C_{1, i}$.

Now we have subdivisions $\mathbf{C}_{1,1}$ of $\mathbf{C}_{1}$ and $\mathbf{C}_{2,1}$ of $\mathbf{C}_{2}$, such that $\phi^{\prime \prime}\left(C_{1,1}^{\prime}\right)=\mathbf{C}_{2,1}^{\prime}$. The subdivision process preserves condition (4) of Definition 4.2. Thus all the conditions of the conclusion of the theorem are satisfied.

I believe that if $M_{1} / G_{1}$ and $M_{2} / G_{2}$ are homeomorphic, then $\left[M_{1}, G_{1}\right]$ and $\left[M_{2}, G_{2}\right]$ are shrink-equivalent. An easy proof of this would simplify the present paper, but I have not been able to find one.

5. The shrink-operation $\alpha$. Given two shrink-equivalent hyperspaces $\left[M_{1}, G_{1}\right]$ and $\left[M_{2}, G_{2}\right]$, a shrink-operation is an operation performed on one or both of them, preserving the relation of shrink-equivalence between them. Such operations will be defined in the following three sections.

Let $\phi$ be a shrink-equivalence between $\left[M_{1}, G_{1}\right]$ and $\left[M_{2}, G_{2}\right]$, with respect to $\mathrm{C}_{1}$ and $\mathrm{C}_{2}$. Now $K_{1}$ is a skew-complex, $\pi_{1} \mid K_{1}$ is skew-simplicial, and $\pi_{1}\left(K_{1}\right)$ is a skew-complex with at most two vertices. Generalizing slightly a definition given in $\S 3$, we say that an element $\pi_{1}^{-1}(x)$ of $G_{1}$ is generic if $x$ lies in the interior of an edge of $\pi_{1}\left(K_{1}\right)$. Let $\sigma^{2} \in K_{1}$, suppose that $\pi_{1}\left(\sigma^{2}\right)$ is an edge of $\pi_{1}\left(K_{1}\right)$, and suppose that if $\pi_{1}^{-1}(x)$ is a generic set intersecting $\sigma^{2}$, then Int $\sigma^{2} \cap \pi_{1}^{-1}(x)$ does not separate $\pi_{1}^{-1}(x)$. Let $B$ be a broken line in $M_{2}$, such that $B^{\prime}=\phi\left(\pi_{1}\left(\sigma^{2}\right)\right)$, and suppose that $B$ intersects $K_{2}$ in at most one or both of the endpoints of $B$. By Theorem 4.3, we may suppose that $\phi$, $C_{1}$, and $C_{2}$ are chosen so that if $X$ is the union of the cells of $C_{2}$ that contain $B$, then $X$ is a 3-cell and $\pi_{2} \mid X$ is a homeomorphism. In the operation $\alpha$, presently to be defined, changes will be made only in $X$, with no changes on the boundary, except for subdivisions. Thus, locally, we are working in a manifold.

Let $C_{1,1}$ and $C_{1,2} \in \mathrm{C}_{1}$, such that $C_{1,1} \cap C_{1,2} \supset \sigma^{2}$; and let $\sigma^{2}=$ $v_{0} v_{1} v_{2}$, as in Figure 1 in $\S 3$. (Note that here and hereafter the notation of simplices is used for skew-simplices, although a skew-simplex is not necessarily determined when its vertices are named.) Let $C_{2, j}(j=1,2)$ be the elements of $\mathbf{C}_{2}$ such that

$$
C_{2, j}^{\prime}=\pi_{2}\left(C_{2, j}\right)=\phi\left(C_{1, j}^{\prime}\right)=\phi\left(\pi_{1}\left(C_{1, j}\right)\right) .
$$

Now split $v_{1} v_{2}$ into two edges $e_{1}, e_{2}$, as in Figure 2. The edge $e_{2}$ now cuts $\sigma^{2}$ into 2-cells. We now subdivide again, like this: 


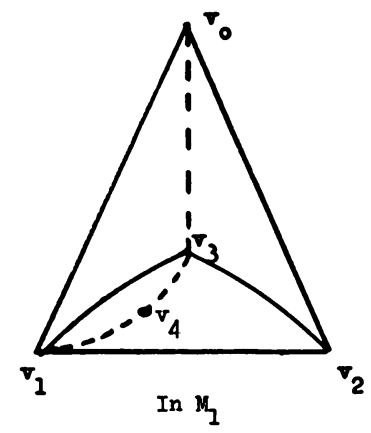

FIGURE 12

Here $v_{3}$ and $v_{4}$ are new vertices, and the three dotted edges are new edges. Thus we have subdivided each set $\mathrm{Bd} C_{1, j}$. We introduce a new vertex $v_{1, j}$ in Int $C_{1, j}$, and subdivide $C_{1, j}$ by forming the join. This gives a cell-decomposition $C_{1,1}$ of $M_{1}$, in which the new singularity complex $K_{1,1}$ forms a subcomplex. Note the purpose of the two new vertices, and the new dotted edges, in $\mathrm{Bd} C_{1, j}$ : we need to subdivide finely enough so that the elements of $\pi_{1}\left(C_{1,1}\right)$ are cells.

We now need to copy all this in $M_{2}$, so as to get a shrink-equivalence between two new hyperspaces. Let $B$ be as in the beginning of this section. We express $B$ as the union of two broken lines $B_{1}$ and $B_{2}$, end to end. We know that $C_{2,1} \cap C_{2,2}=B$. (See Theorem 4.3; we are not distinguishing between sets in $X$ and their projections under $\pi_{2}$.) We now split $C_{2,1} \cup C_{2,2}$ apart, along Int $B_{2}$, getting broken lines $B_{2}^{\prime}, B_{2}^{\prime \prime}$, whose union is a polygon $J$. As before, the dotted edges belong to the subdivision.

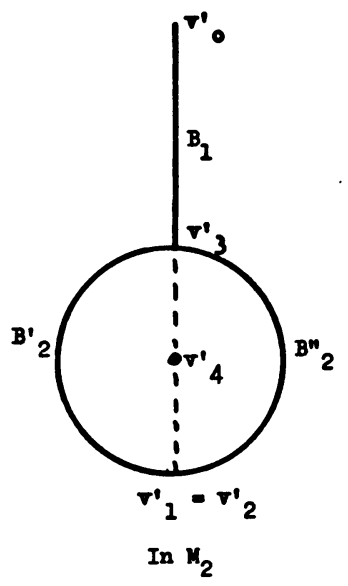

FIGURE 13

We form a new hyperspace $\left[M_{2}, G_{2}^{\prime}\right]$ in which $\pi_{2}^{\prime}(J)$ is a point.

The sets $C_{2, j}$ have now been replaced by 3-cells $E_{2, j}$, intersecting in the union of $B_{1}$ and a 2-cell spanning $J$. In the interior of $E_{2, j}$ we introduce a 
new vertex $v_{2, j}$, and form the join with the given cell-decomposition of $\mathrm{Bd} E_{2, j}$, with identifications along $B_{1}$. This gives a cell-decomposition $C_{2,1}$ of $M_{2}$, in which the new singularity set $K_{2,1}$ forms a subcomplex. In fact, there is a homeomorphism $\phi: M_{1} / G_{1}^{\prime} \leftrightarrow M_{2} / G_{2}^{\prime}$ which is a shrink-equivalence with respect to $C_{1,1}$ and $C_{2,1}$. To construct such a $\phi_{1}$, we use the old $\phi$ on the old sets $C_{1}^{\prime}=\pi_{1}\left(C_{1}\right)$; and on the new sets $C_{1}^{\prime}=\pi_{1}^{\prime}\left(C_{1}\right)\left(C_{1} \in \mathrm{C}_{1,1}\right)$ we follow the directions given by the primes in Figure 13. That is,

$$
\begin{aligned}
& \pi_{1}^{\prime}\left(v_{0} v_{1} v_{3}\right) \leftrightarrow \pi_{2}^{\prime}\left(v_{0}^{\prime} v_{3}^{\prime}\right)=\pi_{2}^{\prime}\left(B_{1}\right) ; \\
& \pi_{1}^{\prime}\left(v_{0} v_{3} v_{2}\right) \leftrightarrow \pi_{2}^{\prime}\left(B_{1}\right) ;
\end{aligned}
$$

the upper edge $\pi_{1}^{\prime}\left(v_{1} v_{3}\right)$ is mapped onto $\pi_{2}^{\prime}\left(v_{3}^{\prime}\right)$, and similarly for the other new edges. The extension of $\phi_{1}$ to the new 2 -faces shown in the figure is obvious. Finally, we define

$$
\phi_{1}\left(\pi_{1}^{\prime}\left(v_{1, j}\right)\right)=\pi_{2}^{\prime}\left(v_{2, j}\right),
$$

and extend $\phi_{1}$ so as to preserve joins.

Thus $\phi_{1}$ is a shrink-equivalence, with respect to $C_{1,1}$ and $C_{2,1}$. The operation $\left[M_{i}, G_{i}\right] \rightarrow\left[M_{i}, G_{i}^{\prime}\right]$, defined in the above discussion, will be called the operation $\alpha_{1}$. Thus we have proved the following.

\section{THEOREM 5.1. $\alpha_{1}$ is a shrink-operation.}

This theorem does not supersede the preceding discussion; the rest of the section uses the latter.

Consider now the 2-faces $v_{1, j} v_{0} v_{3}, v_{1, j} v_{3} v_{4}, v_{1, j} v_{4} v_{1}$ of $\mathbf{C}_{1,1}(j=1,2)$. Their union is a polyhedral disk; see Figure 14.

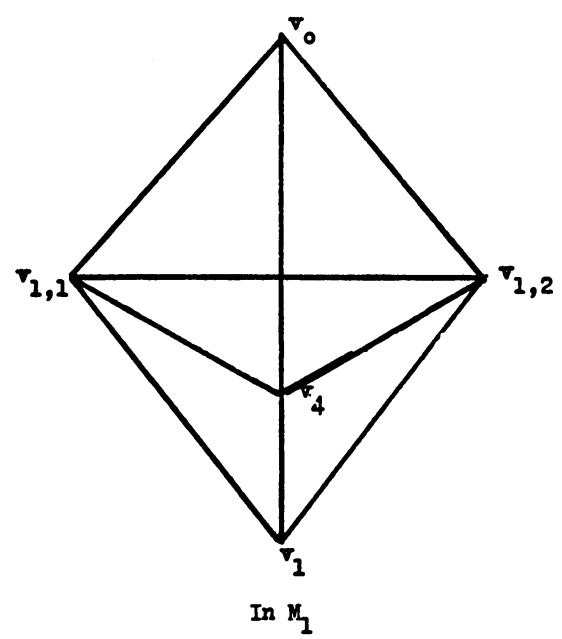

FIGURE 14

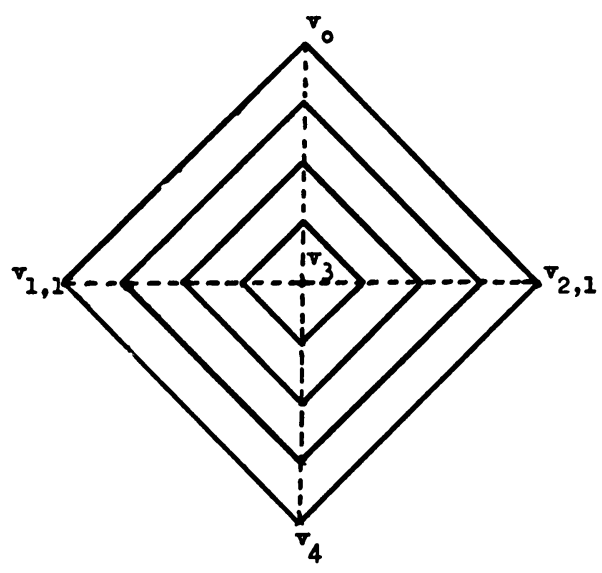

In $M_{1}$

Figure 15 
Let $D_{1}=v_{1,1} v_{0} v_{3} \cup v_{1,1} v_{3} v_{4} \cup v_{2,1} v_{0} v_{3} \cup v_{2,1} v_{3} v_{4}$, so that $D_{1}$ is a polyhedral disk. Adjoining these "simplices," plus their faces, to $K_{1,1}$, we get a new pseudo-simplicial complex $K_{1,2}$. We now define a new hyperspace $G_{1}^{\prime \prime}$, in which $D_{1}$ is mapped onto an edge, as indicated in Figure 15. There is now a subdivision $C_{1,2}$ of $C_{1,1}$, in which $D_{1}$ forms a subcomplex.

All this can be copied in $M_{2}$; the figures would be the same, except for primes on the vertices. Thus we get $D_{2}$ in $M_{2}, K_{2,2}, G_{2}^{\prime \prime}$, and $\mathrm{C}_{2,2}$; and there is a shrink-equivalence between $\left[M_{1}, G_{1}^{\prime \prime}\right]$ and $\left[M_{2}, G_{2}^{\prime \prime}\right]$.

Steps 3 and 4 of $\S 3$ are now mathematically legitimate: first we split $M_{1}$ along Int $D_{1}$, and then we map the resulting 3-cell onto the union of two edges. (See Figures 5 and 6 of $\S 3$.) This gives $\left[M_{1}, G_{1}^{\prime \prime \prime}\right]$. We supposed at the outset that if $\pi_{1}^{-1}(x)$ was a generic set intersecting $\sigma^{2}$, then $\pi_{1}^{-1}(x) \cap \operatorname{Int} \sigma^{2}$ does not separate $\pi_{1}^{-1}(x)$. It follows that Steps 3 and 4 preserve the topology of the hyperspace. And in fact they also preserve the relation of shrink-equivalence. Under the splitting, where $D_{1}$ is replaced by a 3-cell $C$, cells of $C_{1,2}$ go onto cells of a cell-decomposition $\mathrm{C}_{1,3}$ of $M-$ Int $C$; and under the "mashing" operation of Step 4, cells of $\mathbf{C}_{1,3}$ go onto elements of a cell-decomposition $\mathrm{C}_{1,4}$ of $M_{1}$. It is thus a straightforward matter to define a homeomorphism

$$
\begin{gathered}
\phi_{2}: M_{1} / G_{1}^{\prime \prime \prime} \leftrightarrow M_{2} / G_{2}^{\prime \prime}, \\
: \mathbf{C}_{1,4}^{\prime} \leftrightarrow \mathbf{C}_{2,2}^{\prime} .
\end{gathered}
$$

The total operation described in this section is called the operation $\alpha$. We have proved:

THEOREM 5.2. $\alpha$ is a shrink-operation.

Recall that the configuration that we start with, in the operation $\alpha$, is described in Figure 1 of $\S 3$. The final result is described by Figures 4 and 6 .

Let $[M, G]$ be a pseudo-simplicial hyperspace of $M$, with projection $\pi$. In the interior of each edge $e_{i}$ of the skew-complex $\pi(K)$, we choose a point $x_{i}$. Then $\pi^{-1}\left(x_{i}\right)$ is a generic element of $G$. The index Ind $G$ of $G$ is the sum of the 1-dimensional Betti numbers $p^{1}\left(\pi^{-1}\left(x_{i}\right)\right)$, with integers modulo 2 as coefficients. Since $\pi \mid K$ is skew-simplicial, it is evident that Ind $G$ is independent of the choice of the points $x_{i}$. Note also that if Ind $G=0$, then all generic sets are acyclic.

THEOREM 5.3. If $\left[M_{1}, G_{1}\right] \rightarrow\left[M_{1}, G_{1}^{\prime}\right]$ and $\left[M_{2}, G_{2}\right] \rightarrow\left[M_{2}, G_{2}^{\prime}\right]$, under the operation $\alpha$, then Ind $G_{1}^{\prime}=$ Ind $G_{1}-1$.

Proof. Compare Figures 1 and 6 , and recall the initial hypothesis for $\sigma^{2}$. 


\section{The shrink-operation $\beta$.}

THEOREM 6.1. Let $\phi$ be a shrink-equivalence between $\left[M_{1}, G_{1}\right]$ and $\left[M_{2}, G_{2}\right]$, with respect to $\mathbf{C}_{1}$ and $\mathbf{C}_{2}$. Let $\sigma^{2} \in K_{2}$, with two edges $\sigma_{1}^{1}$ and $\sigma_{2}^{1}$, such that $\pi_{2}\left(\sigma^{2}\right)$ is an edge of $\pi_{2}\left(K_{2}\right)$ and $\pi_{2}\left(\sigma_{1}^{1}\right)=\pi_{2}\left(\sigma_{2}^{1}\right)=$ $\pi_{2}\left(\sigma^{2}\right)$. Let $G_{2}^{\prime}$ be the decomposition of $M_{2}$ obtained by splitting $\sigma^{2}$ away from the rest of $K_{2}$ along Int $\sigma_{1}^{1}$. Then there is a pseudo-simplicial hyperspace $\left[M_{1}, G_{1}^{\prime}\right]$ such that (1) $\left[M_{1}, G_{1}^{\prime}\right]$ and $\left[M_{2}, G_{2}^{\prime}\right]$ are shrink-equivalent, and (2) Ind $G_{1}^{\prime} \leqslant$ Ind $G_{1}$.

(For an explicit definition of what we mean by "splitting" operations, see the remarks preceding Definition 4.2.)

DEFinition. The operation described in Theorem 6.1 will be called the operation $\beta$.

Evidently Theorem 6.1 is designed to justify Step 5 of $\S 3$.

Proof. Let $C_{2}$ and $D_{2}$ be the two 3-faces of $\mathrm{C}_{2}$ that have $\sigma^{2}$ as a face, and let $c_{2}$ and $d_{2}$ be the 2-faces of $C_{2}$ and $D_{2}$ (other than $\sigma^{2}$ ) that have $\sigma_{1}^{1}$ as an edge. Let $c_{1}$ and $d_{1}$ be the 2 -faces of $\mathrm{C}_{1}$ such that

$$
\pi_{2}\left(c_{2}\right)=\phi\left(\pi_{1}\left(c_{1}\right)\right), \quad \pi_{2}\left(d_{2}\right)=\phi\left(\pi_{1}\left(d_{1}\right)\right) .
$$

Since $\phi$ is a homeomorphism, it follows that Int $\pi_{1}\left(c_{1} \cup d_{1}\right)$ separates $M_{1} / G_{1}$ locally if and only if Int $\pi_{2}\left(c_{2} \cup d_{2}\right)$ separates $M_{2} / G_{2}$ locally.

Now perform the splitting of $K_{2}$ along Int $\sigma_{1}^{1}$ by splitting $M_{2}$ apart along Int $\left(c_{2} \cup d_{2}\right)$, in the sense explained just before Definition 4.2. Thus $c_{2} \cup d_{2}$ is replaced by the union of two 3-cells $E_{2,1}$ and $E_{2,2}$, intersecting in a common 2-face, where $\sigma_{1}^{1}$ used to be. Thus we have a new pseudo-simplicial decomposition $\mathrm{C}_{2,1}$ of $M_{2}$.

We need to copy this splitting operation, in some way, in $M_{1}$. Let $e$ be the edge of $c_{1}$ such that $\phi\left(\pi_{1}(e)\right)=\pi_{2}\left(\sigma_{1}^{1}\right)$, let $y$ be a point of Int $\pi_{1}(e)$, and let $x_{1}=c_{1} \cap \pi_{1}^{-1}(y)$. Now $\pi_{1}^{-1}(y)$ is a connected linear graph, and so $\pi_{1}^{-1}(y)$ contains a broken line $B$ joining $x_{1}$ to a point $x$ of $d_{1}$. Since $\pi_{1} \mid K_{1}$ is skew-simplicial, it follows that $B$ intersects every $\tau^{2} \in K_{1}$ in a broken line, a point, or the empty set. Let $B_{1}, B_{2}, \cdots, B_{n}$ be the broken lines $\tau^{2} \cap$ $B$, in the order of their appearance on $B$, starting at $x_{1}$. (The $B_{j}$ 's are not necessarily linear intervals, because $K_{1}$ is not in general a complex.) Let the endpoints of the $B_{j}$ 's be $x_{1}, x_{2}, \cdots, x_{n+1}$ (in the order from $x_{1}$ ), so that $x=x_{n+1}$. For each $j$, let $\tau_{j}^{2}$ be the skew-2-simplex of $K$ that contains the broken line $B_{j}=x_{j} x_{j+1}$. For each $j$, let $\tau_{j}^{1}$ be the edge of $K_{1}$ that contains $x_{j}$. Then $\tau_{j}^{1}$ is an edge of $\tau_{j}^{2}$ for each $j$; and if $j>1$, then $\tau_{j}^{1}$ is also an edge of $\tau_{j-1}^{2}$. Since $K_{1}$ is merely a skew-complex, $\tau_{j-1}^{2} \cap \tau_{j}^{2}$ may contain more than $\tau_{j}^{1}$. Note also that nonconsecutive simplices $\tau_{j}^{2}$ may intersect in 
unpredictable ways, on edges which are mapped onto points by $\pi_{1}$. Thus the set

$$
R=\bigcup_{j=1}^{n} \operatorname{Int} \tau_{j}^{2} \cup \bigcup_{j=1}^{n+1} \operatorname{Int} \tau_{j}^{i}
$$

is homeomorphic to the union of an open rectangular region and a pair of opposite faces, but $\bigcup_{j=1}^{n} \tau_{j}^{2}$ is a "singular 2-cell with singularities on its boundary."

We are now ready to "split $M_{1}$ apart along $R$." First we split $M_{1}$ apart along Int $\left(c_{1} \cup \tau_{1}^{2}\right)$. Then we split along Int $\tau_{2}^{2} \cup \operatorname{Int} \tau_{2}^{1}$. Continuing in this fashion, we finally split along $\operatorname{Int}\left(d_{1} \cup \tau_{n}^{2}\right)$. Thus the union of $c_{1}, d_{1}$, and the closure of $R$ is replaced by a 3-cell $C$, with singularities on its boundary, such that the boundary of $C$ is the union of the sets obtained by splitting $c_{1}, d_{1}$, and the simplices $\tau_{j}^{2}$. This gives a new $G_{1}^{\prime}$, and a new $K_{1}^{\prime}$, on which the new $\pi_{1}^{\prime}$ is skew-simplicial. Obviously it does not give a pseudo-simplicial cell-decomposition of $M_{1}$, because $C$ is not a cell. But we can subdivide $C$ into a chain $C_{0}, C_{1}, \cdots, C_{n}, C_{n+1}$ of polyhedral 3-cells, such that the following are true.

(1) For each $i<n+1, C_{j} \cap C_{j+1}$ is a polyhedral 2-cell, bounded by the 1 -sphere obtained by splitting $\tau_{j+1}^{1}$, and intersecting $K_{1}^{\prime}$ nowhere else.

(2) $\mathrm{Bd} C_{0}$ is the union of $C_{0} \cap C_{1}$ and the 2-cells obtained by splitting $c_{1}$.

(3) $\mathrm{Bd} C_{n+1}$ is the union of $C_{n} \cap C_{n+1}$ and the 2-cells obtained by splitting $d_{1}$,

(4) For $0<j<n+1, \mathrm{Bd} C_{j}$ is the union of $C_{j} \cap C_{j-1}, C_{j} \cap C_{j+1}$ and the 2-cells obtained by splitting $\tau_{j}^{2}$.

This gives a pseudo-simplicial cell-decomposition $\mathrm{C}_{1,1}$ of $M_{1}$.

We recall that in $M_{2}, c_{2} \cup d_{2}$ was replaced by two 3-cells $E_{2,1}$ and $E_{2,2}$ with a common 2 -face. To copy the pattern of the chain $C_{0}, C_{1}, \cdots$, $C_{n}, C_{n+1}$ in $M_{2}$, it is sufficient to split apart the common 2-face, $n$ times. This gives a pseudo-simplicial cell-decomposition $\mathrm{C}_{2,2}$ of $M_{2}$.

Obviously Ind $G^{\prime} \leqslant$ Ind $G_{1}$; the splitting operation performed on $K_{1}$ cannot increase the 1-dimensional Betti numbers of the generic sets.

It remains to construct a shrink-equivalence $\phi^{\prime}$ between $\left[M_{1}, G_{1}^{\prime}\right]$ and $\left[M_{2}, G_{2}^{\prime}\right]$. We have a natural correspondence between the faces of $C_{1,1}^{\prime}=$ $\pi_{1}^{\prime}\left(\mathbf{C}_{1,1}\right)$ and those of $\mathbf{C}_{2,2}^{\prime}=\pi_{2}^{\prime}\left(\mathbf{C}_{2,2}\right)$. On each 3-cell $C_{1}^{\prime}$ in $\mathbf{C}_{1,1}^{\prime}$, we can realize this correspondence by a homeomorphism $\phi^{\prime} \mid C_{1}^{\prime}: C_{1}^{\prime} \leftrightarrow C_{2}^{\prime} \in \mathbf{C}_{2,2}^{\prime}$, mapping faces onto faces, in such a way that the mappings agree on common faces of different 3-cells. To do this, we proceed as in the proof of Theorem 4.3, making repeated applications of the theorem which states that every homeomorphism between the boundaries of two cells can be extended to give a homeomorphism between the cells.

So far, the notation $\phi^{\prime} \mid C_{1}^{\prime}$ is an abuse of language, because we have not 
yet defined a global homeomorphism $\phi^{\prime}$ whose restriction to $C_{1}^{\prime}$ is $\phi^{\prime} \mid C_{1}^{\prime}$. But we noted at the outset that $\operatorname{Int}\left(c_{1} \cup d_{1}\right)$ separates $M_{1} / G_{1}$ locally if and only if Int $\left(c_{2} \cup d_{2}\right)$ separates $M_{2} / G_{2}$ locally. As in the preceding discussion, let $e$ be the edge of $c_{1}$ such that $\phi\left(\pi_{1}(e)\right)=\pi_{2}\left(\sigma_{1}^{1}\right)$. Then the edges of $C_{1,1}$, obtained by splitting $e$, are identified by $\pi_{1}^{\prime}$ if and only if the edges of $\mathbf{C}_{2,2}^{\prime}$, obtained by splitting $\sigma_{1}^{1}$, are identified by $\pi_{2}^{\prime}$. Thus the mappings $\phi^{\prime} \mid C_{1}^{\prime}$ fit together to give a well-defined global mapping $\phi^{\prime}$; and by the same reasoning, in reverse, $\phi^{\prime}$ is one-to-one.

7. The shrink-operation $\gamma$. Suppose that $\phi$ is a shrink-equivalence between $\left[M_{1}, G_{1}\right]$ and $\left[M_{2}, G_{2}\right]$, with respect to $\mathrm{C}_{1}$ and $\mathrm{C}_{2}$. Let $\sigma^{2}=v_{0} v_{1} v_{2} \in$ $K_{1}$, such that $\pi_{1}\left(\sigma^{2}\right)$ is an edge of $\pi_{1}\left(K_{1}\right)$; and suppose that $\sigma^{2}$ is free in $K_{1}$, in the sense that

(1) $\pi_{1}\left(v_{0} v_{1}\right)=\pi_{1}\left(v_{0} v_{2}\right)=\pi_{1}\left(\sigma^{2}\right)$, and

(2) $v_{0} v_{2}$. is an edge of no 2 -face of $K_{1}$ other than $\sigma^{2}$.

The operation $\gamma$ deletes Int $\sigma^{2} \cup$ Int $v_{0} v_{2}$ from $K_{1}$. (Similarly if $\sigma^{2} \in$ $K_{2}$, and analogous conditions are satisfied.)

THEOREM 7.1. $\gamma$ is a shrink-operation.

Proof. Let $D$ be a polyhedral 3-cell in $M_{1}$, such that

(1) Int $\sigma^{2} \cup$ Int $v_{0} v_{2}$ lies in Int $D$;

(2) $v_{0} v_{1} \cup v_{1} v_{2}$ lies in $\mathrm{Bd} D$;

(3) If $c$ is a 2 -face of $\mathbf{C}_{1}$, other than $\sigma^{2}$, with $v_{0} v_{2}$ as an edge, then $c \cap D$ is a polyhedral 2-cell $d, d \cap \mathrm{Bd} D$ is a broken line $b$ from $v_{0}$ to $v_{2}$, and Int $b \subset$ Int $c$; and

(4) If $c$ is a 2 -face of $\mathbf{C}_{1}$, and $v_{0} v_{2}$ is not an edge of $c$, then $c$ intersects $D$ at most in a subset of $v_{0} v_{1} \cup v_{1} v_{2}$.

Now the 2-cells $d$ intersect one another only in $v_{0} v_{2}$. We replace each of them, in the 2-skeleton $\mathbf{C}_{1}^{2}$ of $\mathbf{C}_{1}$, by a polyhedral disk $d^{\prime}$ in $D$, such that $\mathrm{Bd} d^{\prime}=b \cup v_{0} v_{1} \cup v_{1} v_{2}$, in such a way that different sets $d^{\prime}$ intersect one another only where they must, in $v_{0} v_{1} \cup v_{1} v_{2}$. Thus each $c \in \mathbf{C}_{1}^{2}$, with $v_{0} v_{2}$ as an edge, is replaced by a 2-cell $c^{\prime}$; and these form the 2-skeleton of a cell-decomposition $D_{1}$ of $M$. In fact, if $\left[M_{1}, G_{1}^{\prime}\right]$ is a new hyperspace, then there is a homeomorphism

$$
\begin{gathered}
\psi: M_{1} / G_{1}^{\prime} \leftrightarrow M_{1} / G_{1}, \\
: \mathrm{D}_{1} \leftrightarrow \mathrm{C}_{1} .
\end{gathered}
$$

(The obvious method works: first we map the projections $\pi_{1}^{\prime}\left(c^{\prime}\right)$ onto the corresponding sets $\pi_{1}(c)$, preserving edges; and then we extend the mapping to the interiors of the 3-cells.) Now $\phi(\psi)$ is a shrink-equivalence $M_{1} / G_{1}^{\prime} \leftrightarrow M_{2} / G_{2}$, $D_{1} \leftrightarrow C_{2}$, which is what we wanted. 
8. Proof of the cloverleaf theorem: weak form. In this section we shall show that under the hypothesis of Theorem 1.1 , there are hyperspaces $S^{3} / K_{1}$ and $M / K_{2}$ which satisfy all the conditions of Theorem 1.1 , except that $K_{1}$ is not necessarily a linear graph. Here the notation of quotient spaces is that of Theorem 1.1. In the following discussion, we resume the notation of the preceding sections.

Let $f$ be a special monotonic mapping $S^{3} \rightarrow M$, let

$$
\begin{aligned}
G_{1} & =\left\{f^{-1}(x) \mid x \in M\right\} \\
& =\left\{\pi_{1}^{-1}(y) \mid y \in S^{3} / G_{1}\right\},
\end{aligned}
$$

and let $K_{1}$ be the singularity complex of $\left[S_{3}, G_{1}\right]$. The corresponding $G_{2}$ is $M$, and $K_{2}$ is empty. By Theorem 4.2, $\left[S^{3}, G_{1}\right]$ and $\left[M, G_{2}\right]$ are shrinkequivalent, under a homeomorphism $\phi$, with respect to cell-decompositions $\mathbf{C}_{1}$ and $\mathrm{C}_{2}$. Hereafter, the conventional notations $G_{i}, K_{i}, \pi_{i}$, and $\mathrm{C}_{i}$ will be used without comment.

In the proof of the weak form of the cloverleaf theorem, we first need to take care of a special case.

Case 1. Suppose that all generic sets $\pi_{1}^{-1}(y)$ are acyclic. Then they all have endpoints, and so they can be eliminated by the operation $\gamma$. This gives $G_{1,1}$ and $K_{1,1}$; and $K_{1,1}$ is the union of two disjoint connected skew-complexes, each of which is mapped onto a point by $\pi_{1,1}$. Thus the weak form of the cloverleaf theorem holds, with $K_{2}=0$.

Case 2. Suppose that some generic set $\pi_{1}^{-1}(y)$ is not acyclic. Then the operation $\alpha$ can be used.

Step A. Perform the operation $\alpha$. This gives hyperspaces $\left[S^{3}, G_{1,1}\right]$ and $\left[M, G_{2,1}\right]$ which are shrink-equivalent, under a homeomorphism $\phi_{1}$, with respect to cell-decompositions $\mathbf{C}_{1,1}$ and $\mathbf{C}_{2,1}$. In $M$, we now have two simply linked polygons $I_{1}, J_{1}$, and spanning disks $D_{1}^{\prime}$ and $E_{1}$, as in Figure 4 of $\S 3$. The new notation is shown in the figure below. $D_{1}^{\prime} \cap E_{1}$ is the broken line $B^{\prime}$.

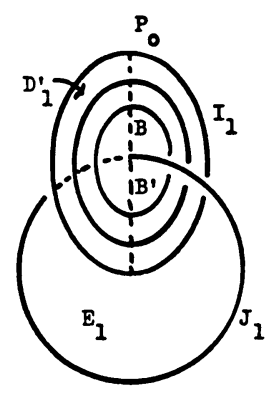

In $M$

FIGURE 16 
$D_{1}^{\prime}$ and $E_{1}$ form subcomplexes of $\mathrm{C}_{2,1}$.

Step B. Let $\sigma^{2}$ be a 2-simplex of $D_{1}^{\prime}$ which contains $B$. Split $\sigma^{2}$ away from Int $B$ by the operation $\beta$, leaving $B$ fixed. This gives new hyperspaces $\left[S^{3}, G_{1,2}\right]$ and $\left[M, G_{2,2}\right]$, which are shrink-equivalent with respect to cell-decompositions $\mathrm{C}_{1,2}$ of $S^{3}$ and $\mathrm{C}_{2,2}$ of $M$.

Step C. By the operation $\gamma$, reduce the generic elements of $G_{2,2}$ to the points of $B$ that contain them, leaving $B$ fixed throughout the process. This gives the situation shown on the left in Figure 10, §3. We now have hyperspaces $\left[S^{3}, G_{1,3}\right]=\left[S^{3}, G_{1,2}\right]$ and $\left[M, G_{1,3}\right]$, and these are shrink-equivalent with respect to $C_{1,3}$ and $C_{2,3}$.

Step D. The disks $D_{1}^{\prime}$ and $E_{1}$ are not necessarily subcomplexes of $C_{2,2}^{2}$, but they still exist; we have

(1) $\mathrm{Bd} D_{1}^{\prime}=I_{1}$;

(2) $\mathrm{Bd} E_{1}=J_{1}$;

(3) $D_{1}^{\prime} \cap E_{1}$ is the broken line $B^{\prime}$;

(4) $D_{1}^{\prime} \cap \pi_{2,3}^{-1} \phi_{3} \pi_{1,3}\left(K_{1,3}\right)=I_{1} \cup B$; and

(5) $E_{1} \cap \pi_{2,3}^{-1} \phi_{3} \pi_{1,3}\left(K_{1,3}\right)$ is the union of $J_{1}$ and the lower endpoint of $B^{\prime}$.

Therefore there is a disk $D_{1}$ such that

(1') $\operatorname{Bd} D_{1}=I_{1}$;

(2) $D_{1}$ and $E_{1}$ are as in the definition of two simply linked cloverleaves; and

(4) $D_{1} \cap \pi_{2,3}^{-1} \phi_{3} \pi_{1,3}\left(K_{1,3}\right)$ is the union of $I_{1}$ and a single point of $J_{1}$.

(To get such a $D_{1}$, we merely move Int $D_{1}^{\prime}$ slightly off to one side, preserving the intersection properties of $D_{1}^{\prime}$ and $E_{1}$.) We now have the following configuration.

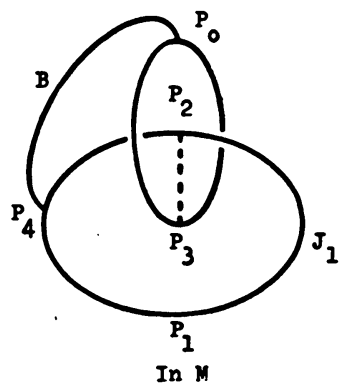

FIGURE 17

In the figure, $D_{1} \cap E_{1}$ is the broken line $P_{2} P_{3}$, and the figure has been distorted so as to convey something of condition ( $\left.4^{\prime}\right)$.

Let $G_{1,4}=G_{1,3}$, and let $G_{2,4}=G_{2,3}$. At this stage, the singularity complex in $M$ is $K_{2,4}=I_{1} \cup J_{1}$, and $B$ intersects this set only at its endpoints. 
By Theorem 4.3 it follows that there are subdivisions $\mathbf{C}_{1,4}$ and $\mathbf{C}_{2,4}$, of $\mathbf{C}_{1,3}$ and $\mathrm{C}_{2,3}$, such that $\left[S^{3}, G_{1,4}\right]$ and $\left[M, G_{2,4}\right]$ are shrink-equivalent with respect to the new subdivisions, and such that if $X$ is the union of the 3-cells of $C_{2,4}$ that contain $B$, then $\pi_{2,4} \mid X$ is a homeomorphism and $X$ is a 3 cell. Therefore Steps $A$ through $D$ can be iterated if need be.

Step E. If all generic sets in $S^{3}$ are acyclic, proceed immediately to Step F. If not, repeat Steps $A$ through $D$ until this condition holds. (The process must terminate, because the operation $\alpha$ reduces the index. See Theorem 5.3.) Then eliminate the generic sets from the singularity complex in $S^{3}$, by iterations of the operation $\gamma$. We then have the following situation.

(1) $S^{3} / G_{1,5}$ and $M / G_{2,5}$ are homeomorphic. (We no longer need shrinkequivalence.)

(2) $K_{1,5}$ contains no generic sets; it has exactly two components $A_{1}, A_{2}$, each of which is mapped onto a point by $\pi_{1,5}$.

(3) We have $I_{1}, I_{2}, \cdots, I_{n}$ and $J_{1}, J_{2}, \cdots, J_{n}$ in $M$, with spanning disks $D_{j}$ and $E_{j}$. These satisfy all the conditions for two simply linked cloverleaves, except that $U I_{j}$ and $U J_{j}$ may be not quite cloverleaves: we have not shown that there is a single point $P$ which is the intersection of every two polygons $I_{j}$; and similarly for the sets $J_{j}$. But $\bigcup I_{j} \cap \bigcup J_{j}=0$; different sets $I_{j}$ (and $J_{j}$ ) intersect only in a single point; $D_{j}$ intersects $D_{k}$ only in $I_{j} \cap I_{k}$ (and similarly for the sets $J_{j}$ and $E_{j}$ ); and $D_{j}$ intersects $E_{k}$ in the way required in the definition of two simply linked cloverleaves. In the figure, we show the case $n=2$.

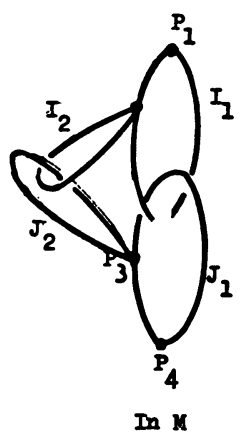

FIGURE 18

To convert such a configuration to the desired final form, we merely map the short arcs $P_{1} P_{2}$ and $P_{3} P_{4}$ onto the points $P_{1}$ and $P_{4}$, by a mapping which is a homeomorphism everywhere else, and which maps the $D_{j}$ 's and $E_{j}$ 's onto polyhedra. (To be precise, the short arcs $P_{1} P_{2}$ (and $P_{3} P_{4}$ ) are the arcs in $I_{1}$ and $J_{1}$ respectively which do not intersect $E_{1}$ (and $D_{1}$ ).) In the general case, iterate this operation $n-1$ times. 
This completes the proof of the weak form of the cloverleaf theorem, with $K_{1}=A_{1} \cup A_{2}$ and $K_{2}=\bigcup I_{j} \cup \cup J_{j}$.

9. Proof of the cloverleaf theorem: conclusion. Let $\left[S^{3}, K_{1}\right]$ and $\left[M, K_{2}\right]$ be as in the weak form of the cloverleaf theorem. We need to reduce $K_{1}$ to a linear graph. For this purpose, we use the unknotting process described in $\S \S 11,12$, and 13 of $[M]$. The following is a sketch. For details, see [M], which gives a complete proof in a more difficult case.

Step 1. First "enlarge" each of the two components $A_{1}$ and $A_{2}$ of $K_{1}$, so that each of them becomes a polyhedral 3-manifold $B_{i}$ with boundary. Let $B=B_{1} \cup B_{2}$. This process can be carried out in such a way that $S^{3} / B$ is homeomorphic to $S^{3} / K_{1}$. (Here quotient spaces are being described in the notation of Theorem 1.1.)

Step 2. Let $W$ be the closure of $S^{3}-B$. By a theorem of R. H. Fox [F], $W$ is homeomorphic to a polyhedron $X$ in $S^{3}$, such that the closure $T$ of $S^{3}-X$ is a tubular set; that is, $T$ is homeomorphic to a regular neighborhood of a linear graph. Thus $S^{3} / B$ and $S^{3} / T$ are homeomorphic.

Step 3. There is now a mapping

$$
\begin{aligned}
& g: S^{3} \rightarrow S^{3} \\
& : T \rightarrow K_{1,1},
\end{aligned}
$$

where $K_{1,1}$ is a linear graph and $g \mid\left(S^{3}-T\right)$ is a homeomorphism. Thus $S^{3} / T$ and $S^{3} / K_{1,1}$ are homeomorphic.

Therefore $S^{3} / K_{1,1}$ and $M / K_{2}$ are homeomorphic, which was to be proved.

10. A strong form of the cloverleaf theorem.

THEOREM 10.1. Under the conditions of the cloverleaf theorem, there are regular neighborhoods $T_{1}$ and $T_{2}$, of $K_{1}$ in $S^{3}$ and of $K_{2}$ in $M$ respectively, such that there is a homeomorphism $\phi: S^{3} / K_{1} \leftrightarrow M / K_{2}$, such that $\phi$ is piecewise linear on every finite polyhedron in $S^{3}-K_{1}$, and $\phi\left(T_{1}\right)=T_{2}$.

In the proof, we shall use the following.

LEMmA 1. There is a homeomorphism $g: S^{3} / K_{1} \leftrightarrow M / K_{2}$ such that $g$ is piecewise linear on every finite polyhedron in $S^{3}-K_{1}$.

Here finite polyhedra and piecewise linearity are defined relative to the given triangulations of $S^{3}$ and $M$, in which $K_{1}$ and $K_{2}$ form subcomplexes.

Proof of lemma. Let $U_{1}=S^{3}-K_{1}, U_{2}=M-K_{2}$. Let $f: S^{3} / K_{1} \leftrightarrow$ $M / K_{2}$ be a homeomorphism. Let $f_{1}=f \mid U_{1}$. Then $f_{1}$ is a homeomorphism $U_{1} \leftrightarrow U_{2}$. For each point $P$ of $U_{1}$, let $\rho(P)$ be half the minimum of (a) the distance from $P$ to $K_{1}$ and (b) the distance from $f(P)$ to $K_{2}$. Then $\rho(P)>0$ 
for every $P ; \rho$ is continuous; and $\rho(P) \rightarrow 0$ as $P \rightarrow K_{1}$. By Theorem 2 of $\left[\mathrm{M}_{5}\right]$, there is a homeomorphism $g_{1}: U_{1} \rightarrow U_{2}$ of $U_{1}$ into $U_{2}$, such that $g_{1}$ is piecewise linear on every finite polyhedron in $U_{1}$ and such that $g_{1}$ is a $\rho$-approximation of $f_{1}$, in the sense that $d\left(g_{1}(P), f_{1}(P)\right)<\rho(P)$ for every $P$. (Here $d$ is the distance function in $M$.)

We now extend $g_{1}$ to get a mapping $g: S^{3} / K_{1} \rightarrow M / K_{2}$. To do this, we define $g(C)=f(C)$, for each of the two components $C$ of $K_{1}$. The resulting function is continuous:

$$
P \approx C \Rightarrow f(P) \approx f(C) \Rightarrow g_{1}(P) \approx f(C) \Rightarrow g(P) \approx g(C) .
$$

Now $g_{1}\left(U_{1}\right)$ is locally Euclidean, and so $g\left(U_{1}\right)$ is open in $M / K_{2}$. If $g\left(U_{1}\right)$ is not all of $U_{2}$, then $g\left(S^{3} / K_{1}\right)$ is not compact, which is impossible. Therefore $g$ is surjective. Therefore $g$ is a homeomorphism $S^{3} / K_{1} \leftrightarrow M / K_{2}$, which was to be proved.

We proceed to the proof of the theorem. Let $L_{2}$ be a component of $K_{2}$. Then the spanning disks of $K_{2}$ that contain the loops of $L_{2}$ intersect only at the intersection point of the loops of $L_{2}$. Therefore $L_{2}$ lies in the interior of a polyhedral disk $D_{2}$ in $M$. Let $T_{2}$ be a regular neighborhood of $K_{2}$, and let $U_{2}$ be the component of $T_{2}$ that contains $L_{2}$. We choose $T_{2}$ sufficiently small so that the set $A_{2}=D_{2} \cap U_{2}$ forms a regular neighborhood of $L_{2}$ in $D_{2}$. Thus $A_{2}$ is a "2-cell with holes," and decomposes $U_{2}$ into two homeomorphic compact pieces $U_{2}^{+}$and $U_{2}^{-}$, such that $U_{2}^{+} \cap U_{2}^{-}=A_{2}$.

There is now a collection $\left\{d_{1}, d_{2}, \cdots, d_{n}\right\}$ of disjoint polyhedral 2-cells in $U_{2}$, such that $d_{i} \cap \operatorname{Bd} U_{2}=\operatorname{Bd} d_{i}$ for each $i$, and such that the $d_{i}$ 's decompose $U_{2}$ into polyhedral 3-cells $C_{j}$ which intersect only in sets $d_{i}$, and such that each $d_{i}$ intersects $A_{2}$ in a linear interval. (The $d_{i}$ 's are "orthogonal to the edges of $L_{2}$ ").

Now let $T_{1}$ be a regular neighborhood of $K_{1}$, chosen sufficiently small so that $g\left(\pi_{1}\left(T_{1}\right)\right) \subset \pi_{2}\left(\right.$ Int $\left.T_{2}\right)$. Let $U_{1}$ be the component of $T_{1}$ such that $g\left(\pi_{1}\left(U_{1}\right)\right) \subset \pi_{2}\left(U_{2}\right)$. Let $S=g\left(\pi_{1}\left(\operatorname{Bd} U_{1}\right)\right)$. We adjust the homeomorphism $g$, if need be, so that $S$ is in general position relative to $A_{2} \cup \cup d_{i}$, in the sense that neither of these sets contains a vertex of the other. It follows that:

(1) for each $i, S \cap d_{i}$ is the union of a finite collection of disjoint (simple closed) polygons;

(2) $S \cap A_{2}$ has the same property; and

(3) for each $i$, the intersections of $S \cap d_{i}$ with $A_{2}$ are "true crossing points" of $S \cap d_{i}$ with $A_{2}$.

Lemma 2. Let $J$ be a polygon in $S$, and suppose that $J$ is contractible in $g\left(\pi_{1}\left(U_{1}\right)\right)-\pi_{2}\left(K_{2}\right)$. Then $J$ bounds a 2-cell in $S$. 
Proof of Lemma. Since $S$ is a retract of $g\left(\pi_{1}\left(U_{1}\right)\right)-\pi_{2}\left(K_{2}\right)$, it follows that $J$ is contractible in $S$; and the lemma follows.

Now let $p$ be the total number of points in all sets of the form $S \cap d_{i} \cap$ $A_{2}$. Let $q$ be the total number of components of all sets of the form $S \cap d_{i} \cap$ $U_{2}^{+}, S \cap d_{i} \cap U_{2}^{-}$, and $S \cap A_{2}$. Hereafter we assume that $g$ is chosen in such a way as to minimize $p$ and $q$, in the stated order.

Lemma 3. No set $S \cap d_{i} \cap U_{2}^{+}$or $S \cap d_{i} \cap U_{2}^{-}$contains a polygon.

Proof of Lemma. Let $J$ be such a polygon. Then $J$ bounds a 2-cell $D_{J}$ in $d_{i}$. We may suppose that $J$ is inmost in $d_{i}$, in the sense that $D_{J}$ contains no point of $S-J$. Obviously $J$ is contractible in $g\left(\pi_{1}\left(U_{1}\right)\right)-\pi_{2}\left(K_{2}\right)$. By Lemma 2 it follows that $J$ bounds a 2-cell $d_{J}^{\prime}$ in $S$. There is therefore a piecewise linear homeomorphism $h: M \leftrightarrow M, d_{J}^{\prime} \leftrightarrow d_{J}$, such that $h \mid\left(d_{i}-d_{J}\right)$ is the identity, and such that $h$ differs from the identity only in a small neighborhood of the 3-cell bounded by $d_{J} \cup d_{J}^{\prime}$. We can now move $d_{J}$ slightly off $d_{i}$ (into the interior of one of the sets $C_{j}$ that contains $d_{i}$ ), by a piecewise linear homeomorphism which differs from the identity only in a small neighborhood of $d_{J}$.

All this is impossible, because it reduces $q$ without increasing $p$.

Lemma 4. Let $B$ be a broken line with endpoints $P$ and $Q$, forming $a$ component of a set $S \cap d_{i} \cap U_{2}^{+}$or $S \cap a_{i} \cap U_{2}^{-}$. Let $P Q$ be the broken line from $P$ to $Q$ in $d_{i} \cap A_{2}$. Then $P Q$ contains the point $d_{i} \cap K_{2}$.

Proof of Lemma. If not, $B$ can be moved into $d_{i} \cap U_{2}^{-}$(or $d_{i} \cap U_{2}^{+}$) by a piecewise linear homeomorphism $M \leftrightarrow M, d_{i} \leftrightarrow d_{i}$ which differs from the identity only in a small neighborhood of the 2-cell in $d_{i}$ bounded by $B \cup P Q$. This is impossible, because it reduces $p$.

LEMma 5. Let $B$ be a component of a set $S \cap C_{i} \cap A_{2}$. Then $B$ is a broken line, and the endpoints of $B$ lie in different 2-cells $d_{j}$ and $d_{k}$ which lie in $\mathrm{Bd} C_{i}$.

Proof of lemma. Obviously $B$ is either a broken line or a polygon. If $B$ were a polygon, then $q$ could be reduced, without increasing $p$; the proof is exactly the same as for Lemma 3 . Therefore $B$ is a broken line. Suppose that the endpoints $P, Q$ of $B$ lie in the same set $d_{j}$. Let $P Q$ be the broken line from $P$ to $Q$ in $d_{j} \cap A_{2}$. Then $B \cup P Q$ bounds a 2-cell $D_{B}$ in $C_{i} \cap$ $A_{2}$. Since $B \cap K_{2}=0$, it follows that $P Q \cap K_{2}=0$ and $D_{B} \cap K_{2}=0$. There is therefore a piecewise linear homeomorphism $\phi: M \leftrightarrow M, A_{2} \leftrightarrow A_{2}$, such that $\phi$ differs from the identity only in a small neighborhood of $D_{B}$, and such that $\phi$ moves $B$ into $A_{2} \cap \operatorname{Int} C_{k}$, where $C_{k}$ is the other 3-cell that contains 
$d_{j}$. This is impossible, because it reduces $p$.

LEMma 6. Let $E$ be a component of a set $S \cap C_{i} \cap U_{2}^{+}$(or $S \cap C_{i} \cap$ $\left.U_{2}\right)$, and let $J$ be a component of $\mathrm{Bd} E$. Then (1) $J$ intersects each component of $\left(C_{i} \cap A_{2}\right)-L_{2}$ in a broken line, joining points of different sets $d_{j}, d_{k}$, and (2) if $d_{j} \subset C_{i}$, then $J \cap d_{j}$ is a broken line, joining points of different components of $\left(C_{i} \cap A_{2}\right)-L_{2}$.

Proof of lemma. Consider the 2-cell $D^{2}$ which is the union of (1) the 2-cell $C_{i} \cap A_{2}$ and (2) the nonempty intersections $C_{i} \cap U_{2}^{+} \cap d_{j}$ (each of which is a 2-cell). Let $Z=D^{2}-L_{2}$. Since $D^{2} \cap L_{2}$ is an acyclic linear graph lying in Int $D^{2}$, it follows that $Z$ is homeomorphic to a half-open plane annulus (i.e., a plane annulus bounded by two concentric circles, minus one component of its boundary). The components of $\left(C_{i} \cap A_{2}\right)-L_{2}$, together with the nonempty sets of the form

$$
\left(C_{i} \cap U_{2}^{+} \cap d_{j}\right)-L_{2},
$$

form a decomposition of $Z$, and these sets appear in a cyclic order around $C_{i} \cap L_{2}$, such that any two sets intersect if and only if they are consecutive. Let the sequence be $e_{1}, e_{2}, \cdots, e_{m}$. By the preceding two lemmas, we know that $J$ "runs straight through" the cyclic sequence $e_{1}, e_{2}, \cdots, e_{m} ; J$ can never reverse its direction. Therefore $J$ does not bound a 2-cell in $Z$. It follows that $J \cup \mathrm{Bd} Z$ is the boundary of a (closed) annulus in $Z$. Therefore any 1 cycle that generates the 1-dimensional homology group $H^{1}(J)$ (with integer coefficients) is homologous on $Z$ to a generator of $H^{1}(\mathrm{Bd} Z)$. It follows that the signed sum of the crossing numbers of $J$ with each set $e_{j} \cap e_{j+1}$ is \pm 1 . Since $J$ cannot reverse its direction, the intersections $J \cap e_{i}$ must be connected, and the lemma follows.

Lemma 7. Let $E$ be as in Lemma 6. Then $\mathrm{Bd} E$ is connected.

Proof of Lemma. Since each component $J$ of $\mathrm{Bd} E$ is contractible in $g\left(U_{1}\right)-L_{2}$, it follows by Lemma 2 that $J$ bounds a disk $D_{J}$ in $S$. Either $D_{J} \supset E$ or $D_{J} \cap E=0$. If $D_{J}=E$, then there is nothing to prove. Suppose, then, that either (a) $D_{J} \supset E$ and $D_{J} \neq E$ or (b) $D_{J} \cap E=0$. Since every component of $\operatorname{Bd} E$ contains a broken line lying in a set $d_{j} \cap U_{2}^{+}$, it follows that $D_{J}$ does not lie in $C_{i}$. Therefore $D_{J}$ contains a polygon $J^{\prime}$, lying in a set $d_{j}$. Suppose that $J^{\prime}$ is inmost in $D_{J}$, in the sense that $J^{\prime}$ bounds a 2-cell $D_{J^{\prime}}$ whose interior intersects no set $d_{k}$. Then $D_{J^{\prime}}$ lies in a single set $C_{m}$, and $J^{\prime}$ bounds a 2-cell $d_{j}\left(J^{\prime}\right)$ in $d_{j}$. Since $D_{J} \cap L_{2}=0$, it follows that $d_{j}\left(J^{\prime}\right) \cap$ $L_{2}=0$. If $J^{\prime} \subset U_{2}^{+}$(or $J^{\prime} \subset U_{2}^{-}$) this contradicts Lemma 3. If $J^{\prime}$ intersects $A_{2}$, then $J^{\prime}$ contains a broken line of the type ruled out by Lemma 4 . 
The proof of the theorem can now be completed by standard "pushing and pulling" methods. Let $W$ be the 3-manifold with boundary such that

$$
\operatorname{Bd} W=S \cup \operatorname{Bd} U_{2} \text {. }
$$

From the preceding lemmas it follows by a straightforward construction that $W$ is the image of the Cartesian product $S \times[1 / 3,2 / 3]$ under a piecewise linear homeomorphism. It follows that there is a closed neighborhood $N$ of $W$ which is the image of $S \times[0,1]$, under a piecewise linear homeomorphism

$$
h: S \times[0,1] \leftrightarrow N,
$$

such that

$$
h(S \times 1 / 3)=\operatorname{Bd} U_{2}, \quad h(S \times 2 / 3)=S .
$$

Therefore there is a piecewise linear homeomorphism

$$
\begin{aligned}
H: M & \leftrightarrow M \\
: S & \leftrightarrow \operatorname{Bd} U_{2} .
\end{aligned}
$$

Let $\phi=H(g)$. Then $\phi\left(U_{1}\right)=U_{2}$.

We go through exactly the same procedure with the other component of $T_{1}$ and the other component of $T_{2}$. This completes the proof of the theorem.

\section{BIBLIOGRAPHY}

[B] R. H. Bing, Conditions under which monotone decompositions of $E^{3}$ are simply connected, Bull. Amer. Math. Soc. 63 (1957), 143. Abstract \#325.

[F] Ross Lee Finney III, Some cellular decompositions and pseudo-isotopic mappings of n-manifolds, Dissertation, University of Michigan, Ann Arbor, Mich., 1961.

[H] John Hempel, Construction of orientable 3-manifolds, Topology of 3-manifolds and Related Topics (Proc. The Univ. of Georgia Inst., 1961), Prentice-Hall, Englewood Cliffs, N. J., 1962, pp. 207-212. MR 25 \#3538.

[L] W. B. R. Lickorish, $A$ representation of orientable combinatorial 3-manifolds, Ann. of Math. (2) 76 (1962), 531-540. MR 27 \#1929.

[M] Edwin E. Moise, A monotonic mapping theorem for simply connected 3-manifolds, Illinois J. Math. 12 (1968), 451-474. MR 37 \#2200.

$\left[\mathrm{M}_{5}\right] \longrightarrow$, Affine structures in 3-manifolds. $\mathrm{V}$. The triangulation theorem and Hauptvermutung, Ann. of Math. (2) 56 (1952), 96-114. MR 14, 72.

CENTRO DE INVESTIGACION DEL INSTITUTO POLITECNICO NACIONAL, MEXICO, D. F.

DEPARTMENT OF MATHEMATICS, QUEENS COLLEGE (CUNY), FLUSHING, NEW YORK 11367 (Current address) 